\title{
Preprocessing among the Infalling Galaxy Population of EDisCS Clusters
}

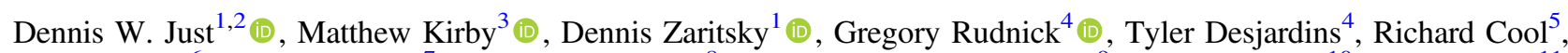 \\ John Moustakas ${ }^{6}$, Douglas Clowe ${ }^{7}$, Gabriella De Lucia ${ }^{8}$, Alfonso Aragón-Salamanca ${ }^{9}$, Vandana Desai ${ }^{10}$ (D), Rose Finn ${ }^{11}$ (DD, \\ Claire Halliday $^{12}$, Pascale Jablonka ${ }^{13,14}$, Justin Mann ${ }^{4}$, Bianca Poggianti ${ }^{15}$ (D) Fu-Yan Bian ${ }^{16}$, and Kelley Liebst ${ }^{17}$ \\ ${ }^{1}$ Steward Observatory, University of Arizona, 933 North Cherry Avenue, Tucson, AZ 85721, USA \\ ${ }^{2}$ Department of Astronomy \& Astrophysics, University of Toronto, $50 \mathrm{St}$ George Street, Toronto, ON M5S 3H4, Canada \\ ${ }^{3}$ Department of Physics, University of Arizona, 1118 E. 4th Street, Tucson, AZ 85721, USA \\ ${ }^{4}$ Department of Physics and Astronomy, University of Kansas, Lawrence, KS 66045, USA \\ ${ }^{5}$ MMT Observatory, 1540 E. Second Street, University of Arizona, Tucson, AZ 85721, USA \\ ${ }^{6}$ Department of Physics \& Astronomy, Siena College, 515 Loudon Road, Loudonville, NY 12211, USA \\ 7 Department of Physics \& Astronomy, Ohio University, Clippinger Labs 251B, Athens, OH 45701, USA \\ ${ }^{8}$ INAF-Osservatorio Astronomico di Trieste, via Tiepolo 11, I-34143, Trieste, Italy \\ ${ }^{9}$ School of Physics and Astronomy, The University of Nottingham, University Park, Nottingham NG7 2RD, UK \\ ${ }^{10}$ IPAC, Mail Code 100-22, Caltech, 1200 E. California Boulevard, Pasadena, CA 91125, USA \\ ${ }^{11}$ Department of Physics, Siena College, Loudonville, NY, USA \\ 12 23, rue d'Yerres, F-91230 Montgeron, France \\ ${ }^{13}$ GEPI, Observatoire de Paris, PSL University, CNRS, 5 Place Jules Janssen, F-92190 Meudon, France \\ ${ }^{14}$ Institute of Physics, Laboratoire d'Astrophysique, Ecole Polytechnique Fédérale de Lausanne (EPFL), Observatoire, 1290 Versoix, Switzerland \\ ${ }^{15}$ INAF-Astronomical Observatory of Padova, vicolo dell'Osservatorio 5, Padova I-35122, Italy \\ ${ }^{16}$ European Southern Observatory, Alonso de Córdova 3107, Casilla 19001, Vitacura, Santiago 19, Chile \\ ${ }^{17}$ School of Earth and Space Exploration, Arizona State University, 781 E. Terrace Mall, Tempe, AZ 85287, USA \\ Received 2018 September 4; revised 2019 September 5; accepted 2019 September 12; published 2019 October 24
}

\begin{abstract}
We present results from a low-resolution spectroscopic survey for 21 galaxy clusters at $0.4<z<0.8$ selected from the ESO Distant Cluster Survey. We measured spectra using the low-dispersion prism in IMACS on the Magellan Baade telescope and calculate redshifts with an accuracy of $\sigma_{z}=0.007$. We find 1763 galaxies that are brighter than $R=22.9$ in the large-scale cluster environs. We identify the galaxies expected to be accreted by the clusters as they evolve to $z=0$ using spherical infall models and find that $\sim 30 \%-70 \%$ of the $z=0$ cluster population lies outside the virial radius at $z \sim 0$.6. For analogous clusters at $z=0$, we calculate that the ratio of galaxies that have fallen into the clusters since $z \sim 0.6$ to those that were already in the core at that redshift is typically between $\sim 0.3$ and 1.5. This wide range of ratios is due to intrinsic scatter and is not a function of velocity dispersion, so a variety of infall histories is to be expected for clusters with current velocity dispersions of $300 \mathrm{~km} \mathrm{~s}^{-1} \lesssim \sigma \lesssim 1200 \mathrm{~km} \mathrm{~s}^{-1}$. Within the infall regions of $z \sim 0.6$ clusters, we find a larger red fraction of galaxies than in the field and greater clustering among red galaxies than blue. We interpret these findings as evidence of "preprocessing," where galaxies in denser local environments have their star formation rates affected prior to their aggregation into massive clusters, although the possibility of backsplash galaxies complicates the interpretation.
\end{abstract}

Key words: galaxies: distances and redshifts - galaxies: evolution - galaxies: interactions

\section{Introduction}

Although a relationship exists between the evolution of galaxies and their environment, as demonstrated by correlations between density and galaxy color (e.g., Hogg et al. 2004), star formation (Lewis et al. 2002; Gómez et al. 2003), and morphology (Dressler 1980; Postman \& Geller 1984), the physical processes that drive these changes and the connection between those processes and environment are not established. While the cores of clusters are the final resting place for quiescent galaxies and are where these trends were discovered, the key to understanding the implicit quenching of star formation and morphological transformation is to study galaxies in the environment where they are being transformed, not where they ultimately reside.

Quenching and morphological transformation do not occur primarily in the cores of clusters, at least not at redshifts $<1$. The decrease in star formation sets in at several virial radii (Lewis et al. 2002; Gómez et al. 2003), and the increase in the S0 fraction since $z \sim 0.5$ (Dressler et al. 1997; Fasano et al. 2000; Desai et al. 2007) is most dramatic in less massive clusters (Poggianti et al. 2009;
Just et al. 2010). Environmentally driven evolution occurs primarily at intermediate densities, which should include the environs outside the cluster virial radius. Such effects are predicted in simulations out to as many as five virial radii (Bahé et al. 2013). Establishing the size and characteristics of the infalling galaxy population will therefore constrain the path to transformation.

As a result of this line of thought, a number of studies have begun to target the outskirts of $z \gtrsim 0.5$ massive clusters (e.g., Moran et al. 2007; Patel et al. 2011; Oemler et al. 2013). However, such studies have been limited to a few clusters $(\sim 10)$, making general conclusions difficult to reach given the variation in properties from cluster to cluster. Because of the high masses of these targeted clusters and correspondingly large virial radii, some of these studies do not probe very far past the virial radius and may miss a significant fraction of the infalling galaxies. Furthermore, such clusters are also rare; hence, the infalling population of more typical clusters has not been explored. This bias may lead to an incomplete picture, given the cluster mass dependence of S0 evolution (Poggianti et al. 2009; Just et al. 2010). 
A fundamental difficulty in studying cluster infalling populations is the contamination of interloping foreground/background galaxies, an issue that becomes more important at larger clustercentric radii, where the relative fraction of interlopers is larger. The studies listed above use spectroscopic redshifts for this purpose, but this approach requires significant telescope time and is thus limited to those few clusters. The alternative approach using photometric redshifts (e.g., Kodama et al. 2001) comes with much lower observational cost, but photometric redshifts are insufficiently precise to securely associate a galaxy with a particular cluster, where $c \delta z \approx 500 \mathrm{~km} \mathrm{~s}^{-1}$ resolution is needed.

We adopt a hybrid approach. We isolate the infalling galaxy population of 21 clusters at $0.4<z<0.8$ using the LowDispersion Prism $\left(\mathrm{LDP}^{18}\right)$ installed in the Inamori-Magellan Areal Camera and Spectrograph (IMACS; Bigelow et al. 1998; Dressler et al. 2006) on the $6.5 \mathrm{~m}$ Magellan Baade telescope. With these data, we measure the number of galaxies these clusters will accrete by $z=0$ to establish how many galaxies may be influenced by the accretion process. We also measure the scatter in this number to estimate the range in accretion histories. We compare models (e.g., Poggianti et al. 2006) that predict the amount of mass accreted by these clusters to our observations. Finally, we measure the optical properties and clustering amplitude of infalling galaxies to quantify the amount of evolution that takes place outside the virial radius.

This paper is organized as follows. In Section 2 we describe our sample selection and the sample's basic properties, and in Sections 3 and 4 we present the imaging and spectroscopic data, respectively. In Section 5 we analyze our clusters using mass infall models and quantify the number of galaxies and optical properties of the infalling population. We conclude in Section 6. All magnitudes in this paper are in the $\mathrm{AB}$ system; to convert these to the Vega system, subtract $0.02,0.06,0.23,0.45$, and 0.55 from the $\mathrm{AB}$ magnitudes for the $B V R I z$ bands, respectively. Throughout the paper, we adopt $H_{0}=70 \mathrm{~km} \mathrm{~s}^{-1} \mathrm{Mpc}^{-1}, \Omega_{0}=$ 0.3 , and $\Omega_{\Lambda}=0.7$, and all cosmology-dependent quantities taken from other studies also use these values. We approximate the virial radii of our clusters as $R_{200}$, the radius inside which the enclosed density is 200 times the critical density of the universe at that redshift.

\section{Sample}

Our sample consists of 21 galaxy clusters. We include 16 of the 20 galaxy clusters in the ESO Distant Cluster Survey (EDisCS; White et al. 2005); see Section 4.1 for details of the four clusters not observed. We also include the seven clusters found serendipitously in this survey. Of these 23 LDP-observed clusters, two are removed from the analysis for reasons given in Section 4.2, resulting in 21 clusters in our final sample. We present basic information on the clusters in Table 1 .

The EDisCS clusters were drawn from candidates in the Las Campanas Distant Cluster Survey (Gonzalez et al. 2001) identified as surface brightness enhancements in the image background. They lie in a band of $\approx 10-14 \mathrm{hr}$ in R.A. and $\approx-13^{\circ}$ to $-11^{\circ}$ in decl. They span a redshift range from $z=0.4$ to 0.8 and cover a spread in velocity dispersion $(\sigma)$ in the range of $\approx 200-1200 \mathrm{~km} \mathrm{~s}^{-1}$ (Halliday et al. 2004; Milvang-Jensen et al. 2008), a wider range of $\sigma$ than other

\footnotetext{
18 Designed by S. Burles for use by the PRIMUS redshift survey (Coil et al. 2011).
}

cluster samples at these redshifts and more representative of the progenitors of $z \sim 0$ clusters (Milvang-Jensen et al. 2008).

We have a variety of data on the cluster cores (the central $\approx 6.5 \times 6.5$ field of view [FOV]), with deep $(I \lesssim 25)$ optical imaging from the FOcal Reducer and low dispersion Spectrograph (FORS2) on the Very Large Telescope (VLT; White et al. 2005), near-infrared (NIR) imaging from the Son OF ISAAC (SOFI) at the New Technology Telescope (White et al. 2005), and optical VLT spectroscopy (Halliday et al. 2004; Milvang-Jensen et al. 2008), weak-lensing maps (Clowe et al. 2006), galaxy morphologies (Desai et al. 2007; Simard et al. 2009), fundamental plane parameters (Saglia et al. 2010), brightest cluster galaxy identifications (Whiley et al. 2008), and MIPSbased star formation rates (Finn et al. 2005, 2010). Wide-field imaging in the mid-infrared with MIPS $\left(\sim 50^{\prime} \times 20^{\prime} \mathrm{FOV}\right)$ and the ultraviolet with Galaxy Evolution Explorer ( $\approx 38^{\prime}$ radius FOV) also exists for cluster subsets but does not appear in this study.

\section{Wide-field Imaging Data}

We use wide-field $\left(\sim 30^{\prime} \times 30^{\prime}\right)$ imaging of our clusters to identify targets for our LDP masks and to measure galaxy magnitudes and colors, which are used for the redshift-fitting portion of the LDP pipeline, as well as for characterizing the galaxies. Our photometry is measured from $B V R I z$ images, with $V R I$ data from the Wide Field Imager (WFI) instrument on the 2.2 m Max Planck Gesellschaft/European Southern Observatory (MPG/ESO) telescope (Baade et al. 1999) and $B z$ data from MOSAIC on the Cerro Tololo Inter-American Observatory (CTIO) Blanco telescope, which have $34^{\prime} \times 33^{\prime}$ and $36^{\prime} \times 36^{\prime}$ FOVs, respectively. Not all clusters have been observed in all five bands. Our entire sample has VRI data, while some clusters appearing in Guennou et al. (2010) have either $B$ or $z$, or both (see Column (9) of Table 1). Details on the imaging data are given below.

\subsection{V-, R-, and I-band Data from WFI}

We reduce the raw images using the techniques described by Clowe \& Schneider (2001, 2002), which involve biassubtracting and flat-fielding each chip separately and removing fringing in the $R$ - and $I$-band images. We calculate astrometric solutions for the images by comparing the image centroids of U.S. Naval Observatory (USNO) reference stars and use the utility imwCS to write a new world coordinate system (WCS) header based on those matches. ${ }^{19}$ This procedure results in an rms position per star of $\approx 0$ !' 3 relative to the USNO coordinates. For Cl1354.2-1230, this method failed to converge, so we define the astrometry using SCAMP (Bertin 2006). The astrometric precision is $\approx 0$ ". 5 for this field.

We create photometric catalogs using SExtractor (Bertin \& Arnouts 1996). We detect sources in the seeing-matched $R$-band image, requiring at least 12 adjacent pixels containing flux $>5 \sigma_{\text {rms }}$ above the background. Photometry is performed in two-image mode for the other bands. Given the wide FOV, we correct for Galactic extinction differentially across the field. The color excess, which is directly proportional to the extinction, across a given field varies by $\approx 0.01-0.02$. We determine $E(B-V)$ at each photometric source using the dust maps of Schlegel et al. (1998) and interpolate the extinction

\footnotetext{
19 Originally written at the University of Iowa, but since adapted and amplified by Jessica Mink at the Smithsonian Astrophysical Observatory (http://tdcwww.harvard.edu/wcstools/imwcs/).
} 
Table 1

LDP-Observed EDisCS Clusters

\begin{tabular}{|c|c|c|c|c|c|c|c|c|c|}
\hline $\begin{array}{l}\text { Field } \\
\text { (1) }\end{array}$ & $\begin{array}{l}\text { Cluster ID } \\
\text { (2) }\end{array}$ & $\begin{array}{l}\text { R.A. } \\
\text { (3) }\end{array}$ & $\begin{array}{l}\text { Decl. } \\
\text { (4) }\end{array}$ & $\begin{array}{c}z \\
(5)\end{array}$ & $\begin{array}{c}\sigma \\
(6)\end{array}$ & $\begin{array}{l}R_{200} \\
\text { (7) }\end{array}$ & $\begin{array}{c}M_{200} \\
(8)\end{array}$ & $\begin{array}{l}\text { Imaging } \\
(9)\end{array}$ & $\begin{array}{c}\text { Seeing }(\operatorname{arcsec}) \\
(10)\end{array}$ \\
\hline 1 & Cl1018.8-1211 & $10: 18: 46.8$ & $-12: 11: 53$ & 0.4734 & $486_{-63}^{+59}$ & $0.93_{-0.12}^{+0.11}$ & $1.53_{-0.52}^{+0.63}(14)$ & $V R I$ & 1.20 \\
\hline 2 & Cl1037.9-1243 & $10: 37: 51.2$ & $-12: 43: 27$ & 0.5783 & $319_{-52}^{+53}$ & $0.58_{-0.09}^{+0.10}$ & $4.06_{-1.68}^{+2.38}(13)$ & $B V R I z$ & 2.10 \\
\hline 3 & Cl1037.9-1243a & $10: 37: 52.3$ & $-12: 44: 49$ & 0.4252 & $537_{-48}^{+46}$ & $1.06_{-0.09}^{+0.09}$ & $2.12_{-0.52}^{+0.59}(14)$ & $B V R I z$ & 2.10 \\
\hline 4 & Cl1040.7-1155 & $10: 40: 40.4$ & $-11: 56: 04$ & 0.7043 & $418_{-46}^{+55}$ & $0.70_{-0.08}^{+0.09}$ & $8.47_{-2.50}^{+3.80}(13)$ & BVRIz & 1.45 \\
\hline 5 & Cl1054.4-1146 & $10: 54: 24.5$ & $-11: 46: 20$ & 0.6972 & $589_{-70}^{+70}$ & $0.99_{-0.12}^{+0.13}$ & $2.38_{-0.75}^{+1.08}(14)$ & $B V R I z$ & 1.20 \\
\hline 6 & Cl1054.7-1245 & $10: 54: 43.6$ & $-12: 45: 52$ & 0.7498 & $504_{-65}^{+113}$ & $0.82_{-0.11}^{+0.18}$ & $1.44_{-0.49}^{+1.21}(14)$ & $B V R I z$ & 1.25 \\
\hline 7 & Cl1059.2-1253 & 10:59:07.1 & $-12: 53: 15$ & 0.4564 & $510_{-56}^{+52}$ & $0.99_{-0.11}^{+0.10}$ & $1.78_{-0.53}^{+0.60}(14)$ & $V R I$ & 1.05 \\
\hline 8 & C11103.7-1245a & 11:03:34.9 & $-12: 46: 46$ & 0.6261 & $336_{-40}^{+36}$ & $0.59_{-0.07}^{+0.06}$ & $4.61_{-1.46}^{+1.65}(13)$ & $B V R I$ & 1.15 \\
\hline 9 & Cl1103.7-1245b & $11: 03: 36.5$ & $-12: 44: 22$ & 0.7031 & $252_{-85}^{+65}$ & $0.42_{-0.14}^{+0.11}$ & $1.86_{-132}^{+1.84}(13)$ & $B V R I$ & 1.15 \\
\hline 10 & Cl1138.2-1133 & $11: 38: 10.3$ & $-11: 33: 38$ & 0.4796 & $732_{-76}^{+72}$ & $1.40_{-0.15}^{+0.14}$ & $5.20_{-1.46}^{+1.69}(14)$ & $B V R I$ & 1.15 \\
\hline 11 & Cl1216.8-1201 & $12: 16: 45.1$ & $-12: 01: 18$ & 0.7943 & $1018_{-77}^{+73}$ & $1.61_{-0.12}^{+0.12}$ & $1.16_{-0.24}^{+0.27}(15)$ & $B V R I$ & 1.20 \\
\hline 12 & Cl1227.9-1138 & $12: 27: 58.9$ & $-11: 35: 13$ & 0.6357 & $574_{-75}^{+72}$ & $1.00_{-0.13}^{+0.13}$ & $2.29_{-0.78}^{+0.97}(14)$ & $B V R I$ & 1.25 \\
\hline 13 & C11227.9-1138a & $12: 27: 52.1$ & $-11: 39: 59$ & 0.5826 & $341_{-46}^{+42}$ & $0.61_{-0.08}^{+0.08}$ & $4.95_{-1.74}^{+2.06}(13)$ & $B V R I$ & 1.25 \\
\hline 14 & Cl1232.5-1250 & $12: 32: 30.5$ & $-12: 50: 36$ & 0.5414 & $1080_{89}^{+119}$ & $1.99_{-0.16}^{+0.22}$ & $1.61_{-0.37}^{+0.59}(15)$ & VRIz & 1.05 \\
\hline 15 & Cl1301.7-1139 & 13:01:40.1 & $-11: 39: 23$ & 0.4828 & $687_{-86}^{+82}$ & $1.31_{-0.16}^{+0.16}$ & $4.29_{-1.42}^{+1.73}(14)$ & $V R I$ & 1.15 \\
\hline 16 & Cl1301.7-1139a & 13:01:35.1 & $-11: 38: 36$ & 0.3969 & $391_{-69}^{+63}$ & $0.78_{-0.14}^{+0.13}$ & $8.32_{-3.67}^{+4.70}(13)$ & $V R I$ & 1.15 \\
\hline 17 & C11353.0-1137 & $13: 53: 01.7$ & $-11: 37: 28$ & 0.5882 & $666_{-139}^{+136}$ & $1.19_{-0.25}^{+0.24}$ & $3.67_{-1.85}^{+2.74}(14)$ & $V R I$ & 1.20 \\
\hline 18 & Cl1354.2-1230 & $13: 54: 09.7$ & $-12: 31: 01$ & 0.7620 & $648_{-110}^{+105}$ & $1.05_{-0.18}^{+0.17}$ & $3.05_{-1.30}^{+1.74}(14)$ & $B V R I z$ & 1.66 \\
\hline 19 & $\mathrm{C} 11354.2-1230 \mathrm{a}$ & $13: 54: 11.4$ & $-12: 30: 45$ & 0.5952 & $433_{-104}^{+95}$ & $0.77_{-0.19}^{+0.17}$ & $1.00_{-0.56}^{+0.82}(14)$ & $B V R I z$ & 1.66 \\
\hline 20 & Cl1411.1-1148 & $14: 11: 04.6$ & $-11: 48: 29$ & 0.5195 & $710_{-133}^{+125}$ & $1.33_{-0.25}^{+0.23}$ & $4.63_{-2.15}^{+2.90}(14)$ & $V R I$ & 1.45 \\
\hline 21 & Cl1420.3-1236 & $14: 20: 20.0$ & $-12: 36: 30$ & 0.4962 & $218_{-50}^{+43}$ & $0.41_{-0.09}^{+0.08}$ & $1.36_{-0.74}^{+0.97}(13)$ & $V R I$ & 1.00 \\
\hline 22 & Cl1103.7-1245 & $11: 03: 43.4$ & $-12: 45: 34$ & 0.9586 & $534_{-120}^{+101}$ & $0.77_{-0.17}^{+0.15}$ & $1.52_{-0.81}^{+1.04}(14)$ & $B V R I$ & 1.15 \\
\hline 23 & C11138.2-1133a & $11: 38: 08.6$ & $-11: 36: 55$ & 0.4548 & $542_{-71}^{+63}$ & $1.05_{-0.14}^{+0.12}$ & $2.14_{-0.74}^{+0.84}(14)$ & $B V R I$ & 1.15 \\
\hline
\end{tabular}

Note. Column (1): cluster field. Column (2): cluster name. Columns (3) and (4): J2000 R.A. (hr) and decl. (deg). Column (5): cluster redshift (Halliday et al. 2004; Milvang-Jensen et al. 2008). Column (6): cluster velocity dispersion in $\mathrm{km} \mathrm{s}^{-1}$ (Halliday et al. 2004; Milvang-Jensen et al. 2008). Column (7): cluster virial radius in Mpc. Column (8): cluster virial mass in units of $M_{\odot}$, with power of 10 in parentheses (using Equation (10) of Finn et al. 2005). Column (9): wide-field imaging bands observed in each field. Column (10): effective WFI seeing after smoothing the images to match the band with the poorest seeing for that cluster.

curve of Cardelli et al. (1989) to the effective wavelength of each bandpass to determine the extinction, assuming $R_{V}=3.1$.

To match the point-spread functions (PSFs) among bands so that aperture-matched magnitudes probe the same region of the galaxy, we smooth the images with a Gaussian kernel selected to match the image with the largest seeing for that field (often the $V$ or $I$ band). The resultant effective seeing is typically $1 . " 2$ (FWHM) for the different fields, except Cl1037.9-1243, which has seeing $\approx 2^{\prime \prime}$. For most clusters, the image quality or effective seeing varies by less than 0 " $1(<0.5$ pixels) over the image; for Cl1227.9-1138, Cl1232.5-1250, Cl1353.0 $-1137, \mathrm{Cl} 1354.2-1230$, and Cl1411.1-1148 it varies $<0$ ". 2 $(<1$ pixel).

The WFI data were taken under nonphotometric conditions and therefore are poorly calibrated. We adjusted the photometric zero-points (ZPs) for the VRI data using well-calibrated and deep VLT images taken as part of the original EDisCS program. To determine the ZPs, we first cross-correlate stars from the WFI images with those from the VLT using a 0.15 matching threshold, resulting in $\sim 20-100$ matches per field. We compare the non-extinction-corrected VLT magnitudes of these matches with their counts in $3^{\prime \prime}$-radius apertures on the WFI images and use linear regression to calculate color terms $\left(a_{\lambda}\right)$ of the following form for each of the bands:

$$
\mathrm{ZP}_{\mathrm{WFI}, \lambda}=\mathrm{ZP}_{\mathrm{VLT}, \lambda}+a_{\lambda}(\text { color })_{\mathrm{WFI}, \lambda},
$$

where (color) is $V-R$ for calibrating the $V$ and $R$ bands and $V-I$ for calibrating the $I$ - band. A first guess for the $V-R$ or
$V-I$ color yields WFI ZPs with which we calculate new VRI magnitudes using

$$
m_{\mathrm{WFI}, \lambda}=-2.5 \log _{10}\left(\text { counts }_{\mathrm{WFI}, \lambda}\right)+\mathrm{ZP}_{\mathrm{WFI}, \lambda} .
$$

These in turn give new $V-R$ or $V-I$ colors. This process is iterated until the WFI magnitude between successive iterations converged to $|\Delta m| \leqslant 0.01 \mathrm{mag}$ or until 20 iterations. Most sources converged within just a few iterations. The final calibration used the median color terms from all clusters, as this value is not expected to vary significantly between observations; however, the normalization of the conversion from the WFI to the FORS photometry was allowed to vary on a clusterby-cluster basis. The uncertainties in VRI ZPs are 0.12, 0.07, and 0.12 , respectively.

$R$-band imaging from the VLT was not available for Cl1018.8-1211, Cl1059.2-1253, Cl1232.5-1250, Cl1301.7 -1139, Cl1353.0-1137, Cl1411.1-1148, and Cl1420.3 -1236 . In what directly follows, all bandpasses refer to the VLT filters. We estimate $R$-band magnitudes from synthetic $R$-band magnitudes that we obtain by fitting the $B V I K$ spectral energy distributions (SEDs) with stellar templates from Hauschildt et al. (1999). We also use this methodology on clusters with $R$-band photometry to assess its accuracy, finding that the absolute value of the difference between predicted and observed mean $R$-band magnitude is $<0.02$. For the seven clusters without VLT $R$-band imaging, we use the $R$-band 
magnitudes derived in this way for the iterated scheme described above when measuring ZPs.

To calculate colors, we use fixed apertures of $1^{\prime \prime}$ radii to maximize signal-to-noise ratio; using a larger aperture introduces more noise into our color measurement. We estimate the total magnitude using the FLUX_AUTO measurement from SExtractor, which fits sources with an ellipse following the method of Kron (1980).

Given the nonstandard method for calibrating our data, we further assessed the quality of our ZP estimates by fitting photometric redshifts using EAZY (Brammer et al. 2008). In some clusters, comparison of the results from an initial pass of EAZY showed severe offsets between the photometric and VLT/FORS2 spectroscopic or preliminary LDP redshifts. The $B$ - and $z$-band data had been taken in photometric conditions and were properly calibrated, so we assumed that these had appropriate ZPs. Many of the redshifts were being skewed toward values that suggested that the VRI filters were the source of the problem. This is unsurprising given the assumptions we made when calibrating the WFI data using the FORS photometry. To account for any offsets that may have been introduced in the VRI photometry by this method, we explored whether small offsets in the photometry could improve the photometric redshift performance. We created a grid of $\mathrm{ZP}$ offsets in the range $-0.2 \leqslant \Delta m \leqslant 0.2$ and looked for the combination of VRI magnitude offsets that minimized the quantity

$$
z_{q}=\frac{\sum_{i=0}^{N}\left(z_{s, i}-z_{p, i}\right)^{2}}{N},
$$

where $z_{s, i}$ is the $i$ th spectroscopic or LDP redshift, $z_{p, i}$ is the $i$ th photometric redshift, and $N$ is the total number of redshifts for a given cluster. The minimization of this $z_{q}$ parameter was performed on a randomly selected subset of half the galaxies, and our shifts were then tested on the remaining half of the spectroscopic sample. These magnitude $\mathrm{ZP}$ corrections result in a median improvement over all clusters in the mean of $\left|z_{s}-z_{p}\right|$ of 0.02 and in the biweight midvariance of $\left|z_{s}-z_{p}\right|$ of 0.015 . For some clusters the improvement in the mean of $\left|z_{s}-z_{p}\right|$ was by as much as 0.06 and in the biweight midvariance of 0.08 .

We estimate photometric errors by placing $10^{3}$ background apertures on each image with radii ranging from 1 to 6 pixels $\left(\approx 0\right.$ !! $\left.2-3^{\prime \prime}\right)$, avoiding sources by using the SEGMENTATION output of SExtractor. We fit the rms fluctuation of counts in each aperture as a function of aperture size to determine the error at $1^{\prime \prime}$ and, for the AUTO magnitudes, at the Kron radius. These errors are typically $\approx 0.01-0.02$ for most of the galaxies that appear in this paper, although at the magnitude limit (see below), the $V$-, $R$-, and $I$-band errors approach $0.03,0.02$, and 0.03 , respectively. Errors on $V-I$ color are $\approx 0.1$ or less.

Comparison with the VLT magnitudes suggests an rms precision that varies with limiting magnitude, in that the rms increases at faint magnitudes. If we only include WFI galaxies with $R_{\text {AUTO }}<23.3$ (our photometric completeness limit; see below), the rms precision is $0.12,0.07$, and 0.12 for the $V, R$, and $I$ total magnitudes, respectively. This includes the Poisson uncertainty of counts in the aperture.

\subsection{B- and z-band Data from MOSAIC}

We use $B$ - and $z$-band data for nine of our clusters obtained by Guennou et al. (2010) with the CTIO Blanco telescope using MOSAIC. These data were reduced with the MIDAS, SCAMP, and SWarp packages (Banse et al. 1988; Bertin et al. 2002; Bertin 2006). Exposure times for the $B$ and $z$ data are $11 \times 600$ s and $18 \times 800 \mathrm{~s}$, respectively. Guennou et al. (2010) describe the data in more detail.

The $B$ - and $z$-band ZPs have errors of 0.09 and 0.07 , respectively (Guennou et al. 2010). The $B$-band ZPs were corrected for galactic extinction using a single $E(B-V)$ value per field from the Schlegel et al. (1998) dust maps; we "decorrect" the $B$-band ZPs so that we can correct each source for extinction individually. After convolving the images to match the largest seeing (often the $V$ or $I$ band), we applied the $B$ - and $z$-band ZPs to their corresponding photometric catalogs generated in two-image mode based on detections in $R$ (see Section 3.1) and correct for extinction differentially using the method described above.

\subsection{Rest-frame Magnitudes and Colors}

In Section 5, we use rest-frame absolute $B$-band magnitudes when selecting galaxies for the analyses, and we also use restframe $U-B$ colors for color-magnitude diagrams (CMDs) and $B-V$ colors to calculate stellar masses in Section 5.3. These restframe magnitudes and colors are calculated using $Q=4 \mathrm{LDP}$ redshifts (see Section 4) with EAZY (Brammer et al. 2008). This code fits the BVRIz photometry (or subset thereof; see Table 1) using linear combinations of a set of theoretical templates that have been reduced to a subset of five "principal component templates" as described in Brammer et al. (2008). These are the same templates used to derive photometric redshifts. In effect the templates serve to interpolate between the observed data points or to extrapolate beyond the bounds of the observed photometry. For some of our lowest-redshift clusters with no $B$-band observations, our bluest observed filter is slightly redward of the redshift restframe $U$-band filter, though always by less than $500 \AA$ for cluster galaxies. Thus, EAZY must slightly extrapolate to measure the $U-B$ color. For these clusters we see no systematic offset in their colors compared to the rest of the systems.

\subsection{Photometric Completeness}

We estimate our magnitude-limited completeness by examining the galaxy number counts as a function of magnitude (Figure 1). Differential number counts with magnitude $(\log d N / d m)$ follow a power-law distribution until the shape of the curve turns over once the catalog starts to become incomplete, with deeper catalogs turning over at fainter magnitudes (e.g., Figure 1 of White et al. (2005), although they applied aperture corrections to their magnitudes that result in a slightly different slope and sharper cutoff at the faint end). In Figure 1, we fit $\log d N / d m$ using a linear regression and find that it follows a power law until $R_{\text {AUTO }} \approx 24.1$, at which point the distribution turns over. The limits for individual fields range from 23.3 to 24.5 with a standard deviation of 0.3 mag. Only one field (Cl1420.3-1236) has a limit brighter than 23.9. To be conservative, we choose a limit for the whole survey that corresponds to that cluster, and thus we are photometrically complete to $R_{\mathrm{AUTO}}<23.3$. 


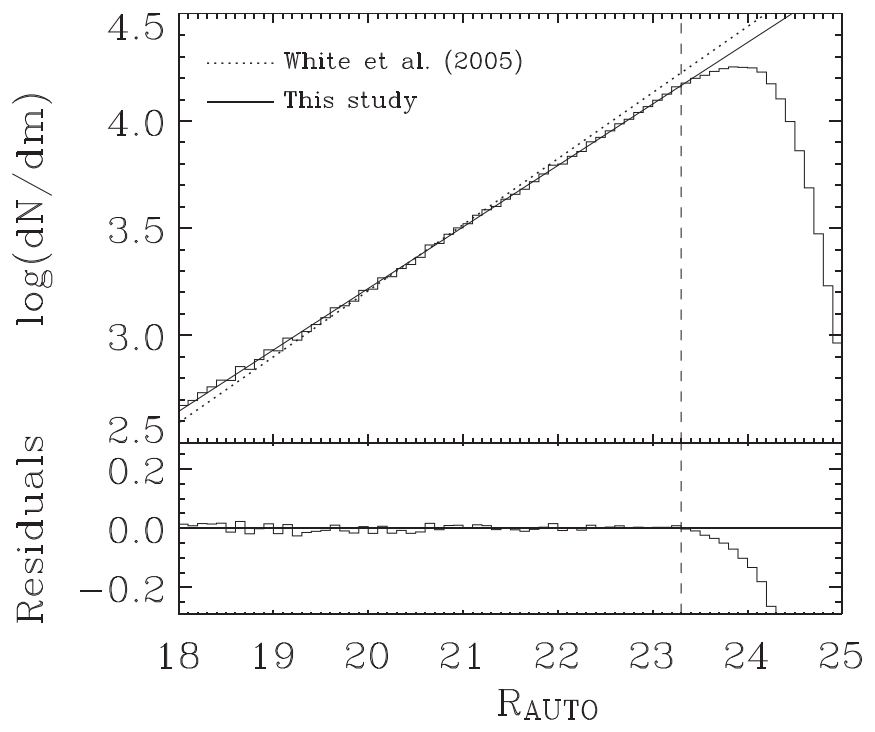

Figure 1. Top panel: differential number counts of $R$-band-detected sources per 0.1 -sized magnitude bin $(d N / d m)$ as a function of $R_{\text {AUTO }}$. We have not removed stars from the distribution. The solid line shows a fit to the distribution using a linear regression, while an estimate of $d N / d m$ for the core VLT photometry from White et al. (2005) is shown as a dotted line. Bottom panel: residuals from the best fit show that $d N / d m$ follows a power law until $R_{\text {AUTO }}=23.3$ (vertical dashed line), a clear sign of incompleteness beyond that magnitude.

\section{LDP Spectroscopic Data}

In this section, we present details on the LDP target selection, as well as the redshift-fitting procedure and results.

\subsection{Target Selection}

We utilize the LDP and the IMACS camera on the Magellan I Baade $6.5 \mathrm{~m}$ telescope at Las Campanas Observatory. This instrument provides spectra with a resolution of $\mathcal{R}=\lambda / \Delta \lambda \approx$ $20-120$ from red to blue wavelengths, an improvement over the resolution achieved with photometric redshifts $(\mathcal{R} \sim 5$ ). The corresponding redshift precision is also improved, as is the overall accuracy. Coil et al. (2011) present more details about the prism and camera characteristics.

Of the original 20 EDisCS fields, four were not targeted with the LDP and so do not appear in this paper. Cl1119-1129 and Cl1238-1144 do not have NIR data; the former contains a $\sigma=166 \mathrm{~km} \mathrm{~s}^{-1}$ cluster, while the latter has only four spectroscopic redshifts at the cluster distance (Milvang-Jensen et al. 2008). Cl1122-1136 does not contain a confirmed cluster, and Cl1202-1224 was not observed owing to the limited telescope time available.

We obtained the LDP data during two observing runs, from 2008 February 7-9 to 2009 March 27-30 (Table 2). Slit dimensions are $1^{\prime \prime} \times 0$ " 8 , compared to $1^{\prime \prime} \times 1$ " 6 for the bulk of the PRIMUS survey (Coil et al. 2011); this choice allows the placing of $\approx 1800-2800$ slits per mask. We chose exposure times of $64 \times 60 \mathrm{~s}$ per mask and used nod-and-shuffle mode to improve sky subtraction.

We observed each field with two masks, except Cl1232 -1250 , which was observed with three masks. Portions of each field are masked out owing to the presence of bright stars. The FOV covers $\sim 0.2 \mathrm{deg}^{2}$ around each cluster, corresponding to clustercentric distances of $\sim 6-8 \mathrm{Mpc}$. Because each mask in a given field has a different center, the final footprint for each field has a nonregular shape.
Table 2

LDP Observing Log

\begin{tabular}{lcc}
\hline \hline Run $^{\mathrm{a}}$ & Cluster & Seeing $(\operatorname{arcsec})$ \\
\hline 1 & Cl1040.7-1155 & 0.7 \\
1 & Cl1054.4-1146 & 2.2 \\
1 & Cl1054.7-1245 & $0.6-1.1$ \\
1 & Cl1103.7-1245 & $0.6-1.0$ \\
1 & Cl1216.8-1201 & 0.7 \\
1 & Cl1227.9-1138 & $0.5-0.9$ \\
2 & Cl1018.8-1211 & $0.6-0.7$ \\
2 & Cl1037.9-1243 & $0.5-1.0$ \\
2 & Cl1059.2-1253 & $0.5-0.6$ \\
2 & Cl1138.2-1133 & $0.5-0.6$ \\
2 & Cl1232.5-1250 & $0.4-0.6$ \\
2 & Cl1301.7-1139 & $0.5-0.7$ \\
2 & Cl1353.0-1137 & $0.4-0.6$ \\
2 & Cl1354.2-1230 & $0.4-0.6$ \\
2 & Cl1411.1-1148 & $0.4-0.7$ \\
2 & Cl1420.3-1236 & $0.5-0.7$ \\
\hline
\end{tabular}

Note.

${ }^{\text {a }}$ Run 1 took place 2008 February 6-8; Run 2 took place 2009 March 27-30.

Table 3

Photometric Targeting Criteria ${ }^{\mathrm{a}}$

\begin{tabular}{lccc}
\hline \hline Cluster & $R_{\text {AUTO,BCG }}$ & Priority \#1 & Priority \#2 \\
\hline Cl1018.8-1211 & 19.64 & $18.64-23.13$ & $\ldots$ \\
Cl1037.9-1243 & 19.79 & $18.79-22.89$ & $\ldots$ \\
Cl1040.7-1155 & 21.17 & $20.17-22.99$ & $18.99-20.17$ \\
Cl1054.4-1146 & 21.20 & $20.20-22.93$ & $18.93-20.20$ \\
Cl1054.7-1245 & 21.09 & $20.09-22.97$ & $18.97-20.09$ \\
Cl1059.2-1253 & 19.20 & $18.20-23.11$ & $\ldots$ \\
Cl1103.7-1245 & 22.87 & $21.87-22.99$ & $18.99-21.87$ \\
Cl1138.2-1133 & 20.03 & $19.03-22.67$ & $\ldots$ \\
Cl1216.8-1201 & 20.56 & $19.56-23.07$ & $19.07-19.56$ \\
Cl1227.9-1138 & 21.06 & $20.06-22.99$ & $18.99-20.06$ \\
Cl1232.5-1250 & 19.12 & $18.12-23.05$ & $\ldots$ \\
Cl1301.7-1139 & 19.56 & $18.56-23.05$ & $\ldots$ \\
Cl1353.0-1137 & 20.29 & $19.29-23.08$ & $19.08-19.29$ \\
Cl1354.2-1230 & 21.27 & $20.27-22.97$ & $18.97-20.27$ \\
Cl1411.1-1148 & 20.79 & $19.79-23.08$ & $\ldots$ \\
Cl1420.3-1236 & 20.09 & $19.09-22.95$ & $\ldots$ \\
\hline
\end{tabular}

Notes. Priority \#1 corresponds to $R_{\mathrm{AUTO}, \mathrm{BCG}}-1<R_{\mathrm{AUTO}} \lesssim 23$; priority \#2 corresponds $19 \lesssim R<R_{\mathrm{AUTO}, \mathrm{BCG}}-1$, when $R_{\mathrm{BCG}, \mathrm{AUTO}}>19$.

${ }^{a}$ All $R_{\text {AUTO }}$ magnitudes in this table are from the photometry on hand when the data were taken. The photometry has since been revised with changes $<0.5$ mag and typical changes of $\approx 0.2$ mag.

There are $(1-2) \times 10^{4}$ sources in our WFI catalog within each LDP footprint. Of these, we target $\approx 3000-5000$ objects per field with the LDP $(\approx 20 \%$ of potential targets, although the percentage ranges among the fields from $15 \%$ to $40 \%$ ). Galaxies are targeted depending on their $R$-band magnitude relative to the brightest cluster galaxy (BCG). Priority \#1 targets have $R_{\mathrm{AUTO}, \mathrm{BCG}}-1<R_{\mathrm{AUTO}} \lesssim 23$. After targeting those, additional slits (priority \#2) are placed on sources with $19 \lesssim R_{\text {AUTO }}<R_{\text {AUTO,BCG }}-1$. In cases where $R_{\mathrm{BCG}, \mathrm{AUTO}}<$ 20 , there are no priority \#2 targets. Finally, we place slits on any galaxies that do meet these criteria but are capable of being targeted in that mask; these "filler" slits are $\sim 10 \%$ of the total. The $R$-band ranges of nonfiller targets per field appear in Table 3. 


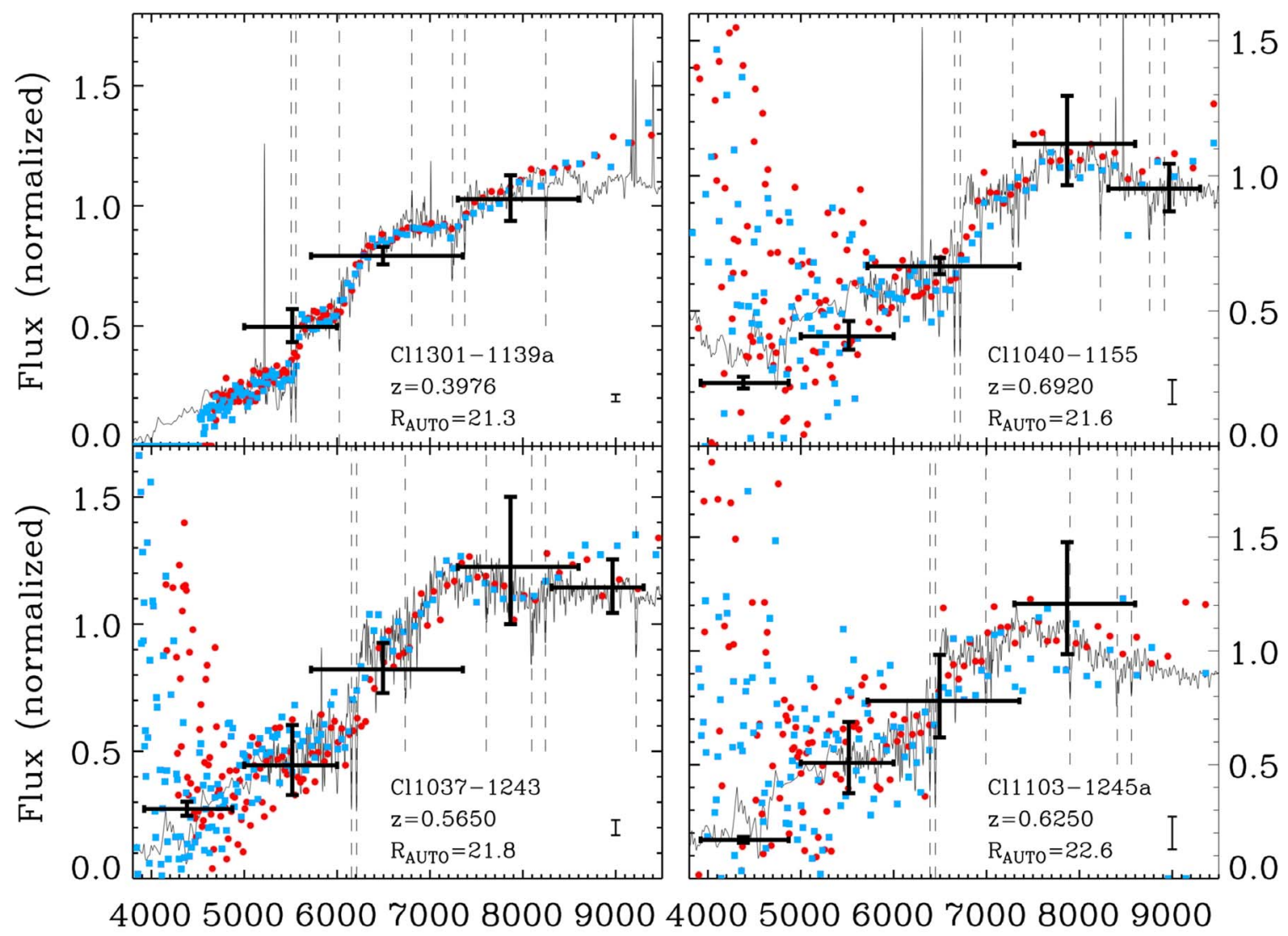

Observed Wavelength $(\AA)$

Figure 2. Sample LDP spectra with $Q=4$ for galaxies in four clusters, spanning $21.3<R_{\mathrm{AUTO}}<22.6$ and $<0.4<z<0.7$. Cyan squares and red circles are the LDP spectra values in each slit of the nod and shuffle, while the continua spectra shown are those of the best-fit templates. The spectra have been normalized to unity at $6800 \AA$ A. Typical errors on the LDP data are shown at the bottom right of each panel. Also plotted are magnitudes (VRI, BVRIz, BVRIz, and BVRI, respectively), and prominent spectral features are shown as vertical dashed lines. The LDP redshifts agree with those measured from FORS2 to $\delta z=0.0024,-0.0132,-0.0139$, and 0.0012 .

The mean separation between adjacent slits for an individual mask is $\approx 20^{\prime \prime}$, with a minimum separation of $10^{\prime \prime}$. However, multiplexing done with multiple masks per field increases the sampling density, with a mean separation of $\approx 15^{\prime \prime}$ and $15 \%$ $20 \%$ of slits separated by $<10^{\prime \prime}$ (with the closest pairs $\approx 1^{\prime \prime}$ apart).

\subsection{LDP Redshifts}

The PRIMUS reduction pipeline simultaneously fits the spectral and photometric data to a set of galaxy templates at different redshifts and calculates a best-fit $\chi^{2}$ value at each redshift (Cool et al. 2013). While the relative astronometry of our observing masks was accurate, they were mildly offset in absolute astronometry. We calculated a new astrometric solution for the WFI imaging after the LDP slit positions were determined by cross-correlating the WFI catalog with the slits using a $1^{\prime \prime}$ matching threshold. The pipeline treats photometric data similarly to 1 pixel of the spectrum. The $\chi^{2}$ fit is determined primarily from the LDP data, but the photometric data help distinguish between redshift solutions. On the basis of the $\chi^{2}$ distribution, both a best-fit redshift and a redshift confidence parameter, $Q$, are calculated. The confidence parameter is assigned using the ratio of the width of the primary peak in the $P(z)$ distribution and the goodness of fit between the first and second peaks. This ratio is then used to assign an integer confidence parameter between 2 and 4, with $Q=4$ objects typically having a narrow primary peak compared to other features in the $P(z)$ distribution. Further details on the redshift-fitting procedure appear in Cool et al. (2013). Example spectra of four cluster galaxies appear in Figure 2.

For objects with more than one redshift measured (given the multiple masks), we take the redshift with the higher-quality flag, $Q$. In cases where there are multiple redshifts with the same $Q$, we randomly select one. This is done to avoid averaging significantly discrepant redshifts when they exist (see Figure 3 for outlier rates).

We define cluster membership as galaxies that have a $Q=4$ best-fit redshift within $\pm 0.02\left( \pm 6000 \mathrm{~km} \mathrm{~s}^{-1}\right)$ of the cluster redshift, which is approximately three times the accuracy of the LDP redshifts (see Section 4.2.1). We choose a fixed cut in redshift, rather than a multiple of the cluster $\sigma$, because the LDP uncertainty is larger than any velocity dispersion in our sample. We do not make any spatial cuts because we are interested in galaxies at large clustercentric radii. This selection results in 1763 galaxies that we place in the cluster environment.

A summary of the number of LDP targets, redshifts, and cluster members is presented in Table 4. Two of the LDPobserved clusters do not appear in this study. Cl1103.7-1245, at $z=0.95$, with only three LDP-selected cluster members, does not have enough cluster members for a meaningful 

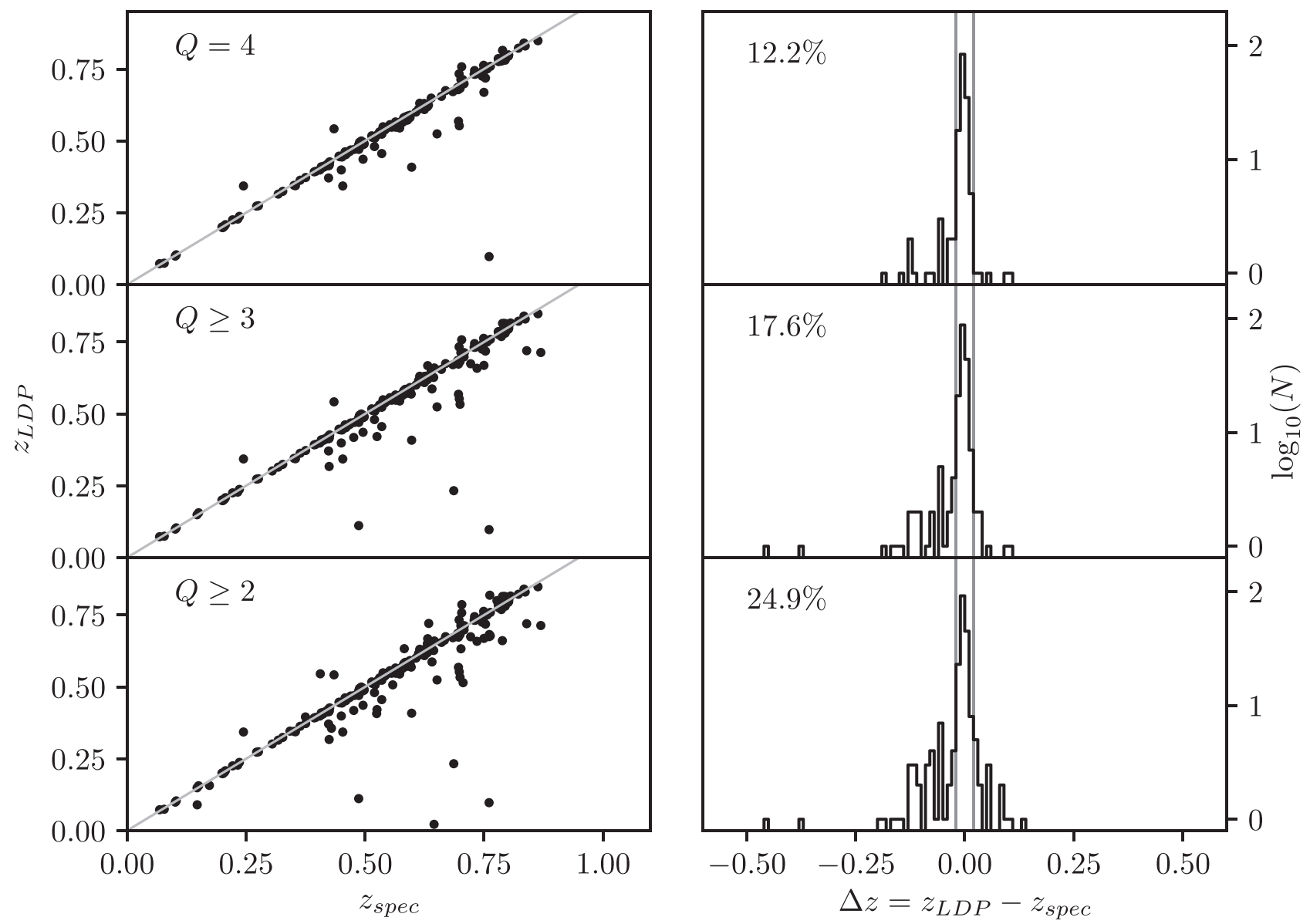

Figure 3. Left panels: comparison of LDP-derived redshifts $\left(z_{\text {LDP }}\right)$ with spectroscopic redshifts $\left(z_{\text {SPEC }}\right)$. The LDP redshifts are split showing different cuts in the quality flag, $Q$. The outliers in $z_{\mathrm{LDP}}$ systematically underestimate the redshift. Right panels: histograms of the residuals from the left panels. Vertical lines show \pm 0.02 , which is the size of the redshift interval used in selecting cluster galaxies. Percentages in the upper left corner show the fraction of outliers outside this interval. The accuracy with the $z_{\mathrm{LDP}}(\sigma=0.007$ for $Q=4)$ is an order-of-magnitude improvement over the photometric redshifts.

analysis. Cl1138.2-1133a, at $z=0.4548$, lies too close to the

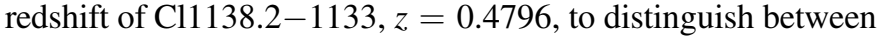
members using the $z_{\text {clus }} \pm 0.02$ selection. We therefore include the latter cluster, with the caveat that some contamination may come from galaxies belonging to the former. We note that C11138.2-1133 is not a significant outlier in any of the analyses that follow.

\subsubsection{LDP Redshift Accuracy}

We assess the accuracy of our LDP-derived redshifts $\left(z_{\mathrm{LDP}}\right)$ by comparing them to the subset of 427 galaxies also observed with VLT/FORS2 $\left(z_{\mathrm{SPEC}}\right)$ over a wide range of redshifts and with photometric redshifts $\left(z_{\mathrm{PHOT}}\right)$ calculated in Pelló et al. (2009) from $B V I K, B V I J K$, and VRIJK imaging of the cluster cores; these filter combinations were chosen based on the initial redshift estimate of the cluster. We match galaxies within $1^{\prime \prime}$ and show the results of these comparisons in Figure 3. We only consider galaxies with $z_{\mathrm{LDP}}<0.85$, which is just above our highest-redshift cluster (Cl1216.8 -1201 at $z=0.79)$; considering the full range of redshifts that PRIMUS fits (out to $z=1.2$ ) affects neither the accuracy nor the outlier rate significantly.

The LDP-derived redshifts are more precise than the photometric redshifts by an order of magnitude, their rms being $\sigma\left(\left|z_{\mathrm{LDP}}-z_{\mathrm{SPEC}}\right|\right)=0.007$ for $Q=4$ data, compared to 0.08 for the photometric redshifts. The outlier rates of LDP-derived redshifts, defined as $\left|z_{\mathrm{LDP}}-z_{\mathrm{SPEC}}\right|>0.02$, depend on the quality cut and range from $25 \%(Q \geqslant 2)$ to $18 \%(Q \geqslant 3)$ to $12 \%(Q=4)$.

In Figure 4 we plot the LDP redshift accuracy and outlier rate dependence on $R$-band magnitude. The precision between the LDP redshifts and true spectroscopic redshifts is constant, even at faint magnitudes for the high-quality $Q=4$ spectra. Above $R_{\text {AUTO }}>21$, we see a large increase in the number of $Q=2$ and 3 spectra, which results in a significant increase in outlier rate. The outlier rate is constant with magnitude for spectra of a given $Q$ value. We do not find any significant dependence of the redshift accuracy on $V-I$ color (Figure 5). The objects with $Q=4$ redshifts make up most of our redshift catalog and have a stable outlier fraction of $12 \%$.

From Figure 3 and 4, it is apparent that the outliers in LDP redshift are skewed toward lower-redshift values (i.e., the LDP fits a lower redshift than the "true" one). Although the outlier rate does not depend on galaxy color, we find a significant dependence on [O II] emission. Being relatively close to the Balmer break, the blending of the two spectral features "drags" the break to a lower redshift, consistent with the bias evident in Figure 3. When we consider $Q=4 \mathrm{LDP}$ spectra, we find that galaxies with $[\mathrm{O}$ II] equivalent widths (EWs) of $\sim 5 \AA$ have an outlier rate of $35 \%$, almost triple the outlier rate for all galaxies (12\%). Moreover, at both higher and lower EWs, the outlier rate drops. This can be understood as galaxies with weaker [O II] emission not suffering from this blend "dragging" the 
Table 4

LDP Information

\begin{tabular}{lccccc}
\hline \hline $\begin{array}{l}\text { Cluster } \\
(1)\end{array}$ & $\begin{array}{c}N_{\text {phot }} \\
(2)\end{array}$ & $\begin{array}{c}N_{\text {targets }} \\
(3)\end{array}$ & $\begin{array}{c}N_{\text {LDP }} \\
(4)\end{array}$ & $\begin{array}{c}N_{Q=4} \\
(5)\end{array}$ & $\begin{array}{c}N_{\text {memb }} \\
(6)\end{array}$ \\
\hline Cl1018.8-1211 & 5349 & 1645 & 1425 & 781 & 86 \\
Cl1037.9-1243 & 10380 & 2231 & 1977 & 1194 & 47 \\
Cl1037.9-1243a & $\ldots$ & $\ldots$ & $\ldots$ & $\ldots$ & 189 \\
Cl1040.7-1155 & 9597 & 2647 & 2218 & 962 & 31 \\
Cl1054.4-1146 & 6297 & 2710 & 2337 & 1174 & 45 \\
Cl1054.7-1245 & 9710 & 2509 & 2070 & 1076 & 89 \\
Cl1059.2-1253 & 9341 & 2275 & 2135 & 1458 & 154 \\
C11103.7-1245a & 9392 & 2561 & 1479 & 584 & 23 \\
Cl1103.7-1245b & $\ldots$ & $\ldots$ & $\ldots$ & $\ldots$ & 16 \\
Cl1138.2-1133 & 6088 & 1530 & 1406 & 1143 & 84 \\
Cl1216.8-1201 & 9435 & 2557 & 2086 & 1022 & 44 \\
Cl1227.9-1138 & 9048 & 2612 & 1983 & 1238 & 86 \\
Cl1227.9-1138a & $\ldots$ & $\ldots$ & $\ldots$ & $\ldots$ & 105 \\
C11232.5-1250 & 9947 & 2455 & 2227 & 1597 & 166 \\
Cl1301.7-1139 & 9426 & 1683 & 1497 & 1085 & 131 \\
Cl1301.7-1139a & $\ldots$ & $\ldots$ & $\ldots$ & $\ldots$ & 158 \\
Cl1353.0-1137 & 11425 & 2222 & 1951 & 1242 & 39 \\
Cl1354.2-1230 & 9483 & 2269 & 2040 & 1468 & 38 \\
Cl1354.2-1230a & $\ldots$ & $\ldots$ & $\ldots$ & $\ldots$ & 80 \\
Cl1411.1-1148 & 10485 & 1897 & 1674 & 1157 & 76 \\
Cl1420.3-1236 & 10103 & 1477 & 1318 & 841 & 76 \\
\hline Total & 145506 & 35280 & 29823 & 18022 & 1763 \\
\hline
\end{tabular}

Note. All numbers only include galaxies brighter than $R<22.9$, our spectroscopic completeness limit. Numbers for Columns (2)-(5) for serendipitously discovered clusters are suppressed, as they are in the same field as the primary cluster. Column (1): cluster name. Column (2): number of photometric sources. Column (3): number of LDP targets. Column (4): number of successfully extracted LDP spectra. Column (5): number of $Q=4$ LDP spectra. Column (6): number of cluster members (defined by $z_{\text {clus }} \pm 0.02$ ).

Balmer break to a lower redshift, while galaxies with stronger [O II] emission have lines that become the dominant redshift feature. Therefore, we are more likely to miss cluster galaxies that have modest [O II] emission at an outlier rate twice as high as for the full galaxy population, or $\sim 4 \%$ of the cluster sample, and have some enhanced contamination from field galaxies with modest [O II] emission at higher redshift.

\subsubsection{Spectroscopic Completeness}

Figure 6 shows the distribution of $R_{\mathrm{AUTO}}$ (analogous to Figure 1) for the LDP targets with successfully measured redshifts. The different curves show the distributions for $Q=2,3$, and 4 redshifts. At the brightest magnitudes, the vast majority of redshifts have a secure $Q=4$ flag. However, at $R_{\text {AUTO }} \gtrsim 20$, redshifts with lower $Q$ flags begin to appear in significant numbers. For all $Q$ values, the distribution turns over before $R_{\text {AUTO }} \sim 23$; our photometric catalog is therefore complete to fainter magnitudes than our spectroscopic one. The full distribution (including all $Q$ values) departs from a power law at $R_{\text {AUTO }} \approx 22.9$, which we take as the estimate of our spectroscopic completeness.

\subsubsection{Radial Completeness}

We also quantify the percentage of successfully measured redshifts as a function of clustercentric distance. We consider the fraction of successfully measured redshifts relative to the number of photometric sources, restricting both to galaxies brighter than our spectroscopic completeness $\left(R_{\mathrm{AUTO}}<22.9\right)$, as a function of

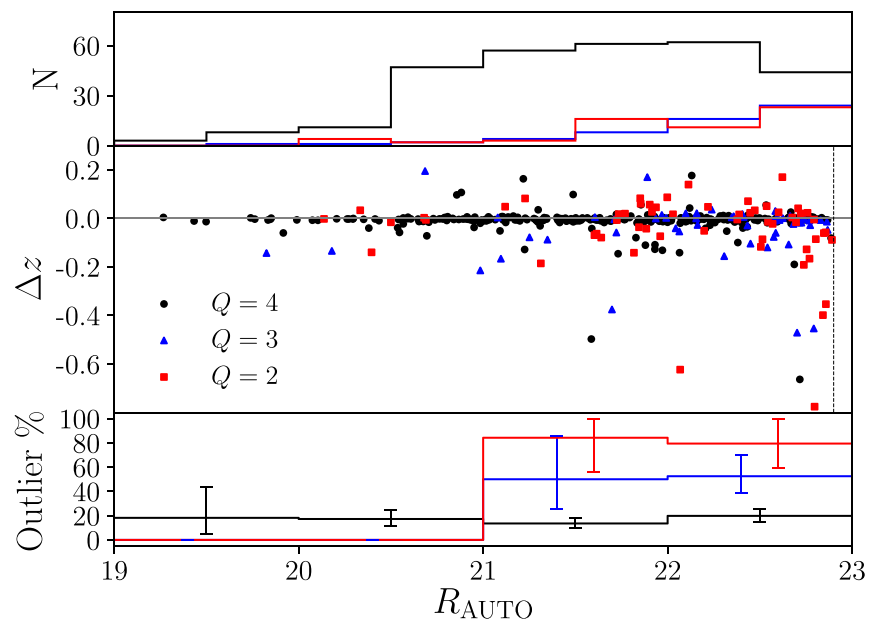

Figure 4. Middle panel: residuals between LDP and FORS2 redshifts as a function of $R$-band magnitude for $Q=4$ (black circles), $Q=3$ (blue triangles), and $Q=2$ (red squares) redshifts. Top panel: histograms of $R$ band magnitude and $z_{\mathrm{LDP}}-z_{\mathrm{SPEC}}$ residuals. Bottom panel: outlier rate $(|\Delta z|>0.02)$ as a function of $R$-band magnitude. The outlier rate is approximately flat for a given $Q$ flag for bins containing more than five objects. The vertical dotted line is our spectroscopic completeness limit.

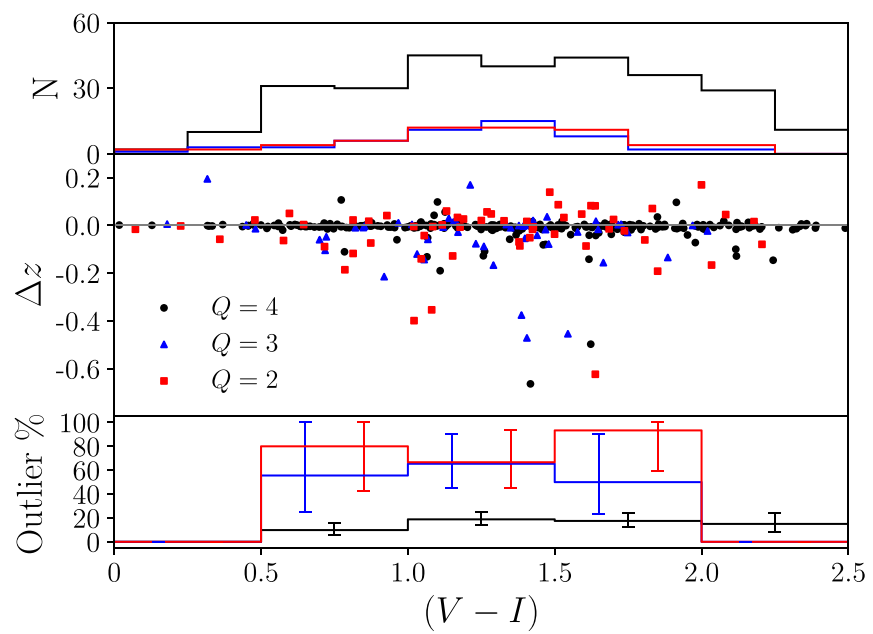

Figure 5. Middle panel: residuals between LDP and FORS2 redshifts as a function of $V-I$ color. Symbols are the same as in Figure 4. Top panel: histogram of $V-I$ color. Bottom panel: outlier fraction as a function of $V-I$ color. The outlier rate is approximately flat for a given $Q$ flag for bins containing more than five objects.

angular distance from the cluster $\left(d_{\text {clus }}\right)$. Figure 7 shows that the percentage of targets with measured redshifts is $\sim 25 \%-35 \%$, depending on $Q$-cut, out to $\sim 10^{\prime}$. Converting this to a physical distance for our typical clusters puts the drop-off at $\sim 4 \mathrm{Mpc}$. The percentage then drops off, as most fields have only one mask coverage at these radii, and approaches zero smoothly rather than abruptly because the offset placement of masks leads to an edge that is not spherically symmetric about the cluster.

Given the surface density of slits on the sky, we are also relatively insensitive to close pairs. While multiplexing with two to three masks per field allows us to measure redshifts for galaxies $\sim 1^{\prime \prime}$ apart that are not near the edges of the footprint, this distance is small compared to the average separation between adjacent slits $\left(\approx 20^{\prime \prime}\right)$. Only $\approx 10 \%$ of slits have separations of $10^{\prime \prime}$ or less. Compared to frequency of slits with separations of $10^{\prime \prime}-30^{\prime \prime}$, the frequency of slits separations $<10^{\prime \prime}$ 


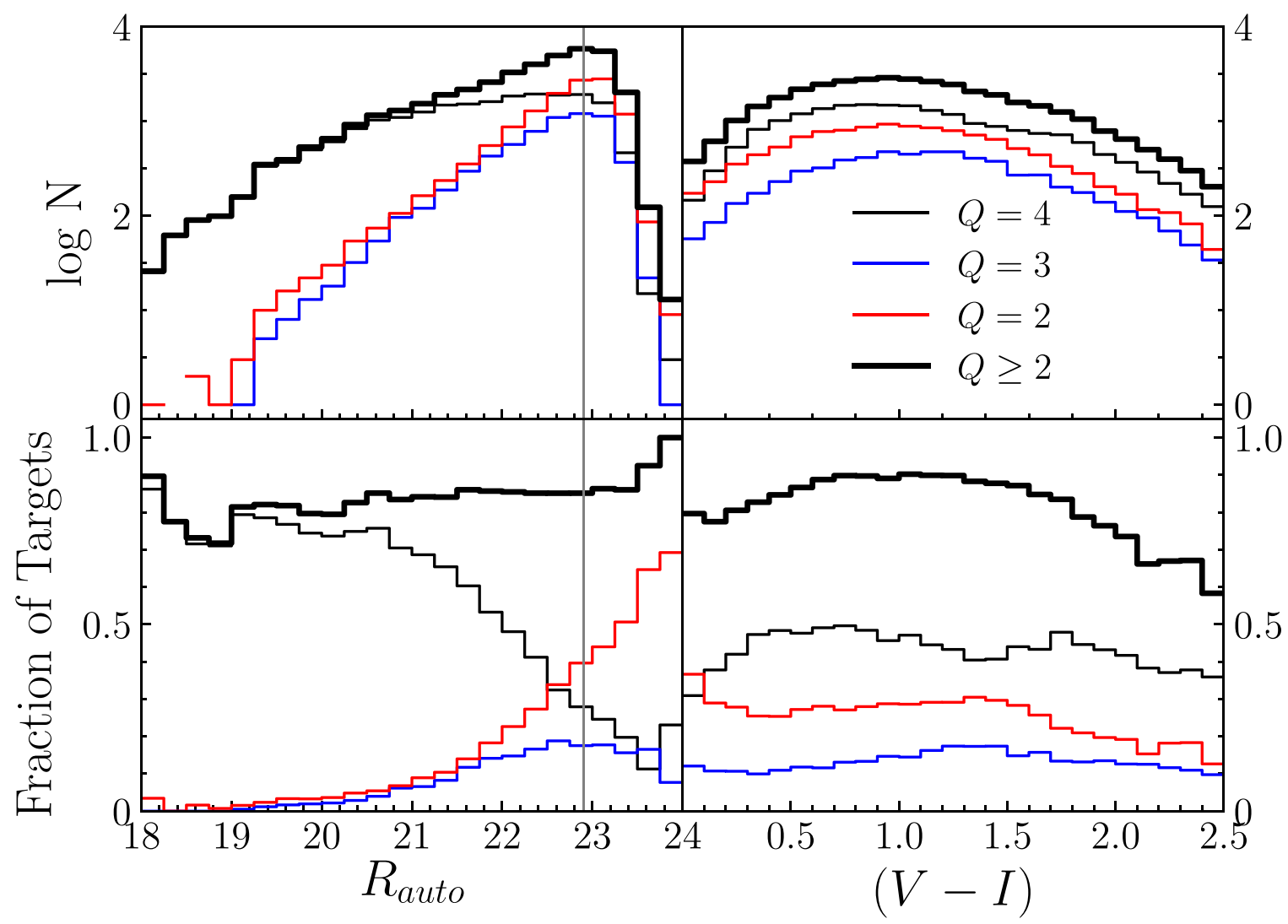

Figure 6. Top left panel: histogram of $R$-band magnitudes for LDP-targeted galaxies with bin sizes of 0.1 for different values of $Q$. The spectroscopic completeness limit at $R_{\text {AUTO }}=22.9$ is shown as a vertical line. Bottom left panel: fraction of targets with a successfully extracted spectrum, i.e., number of spectra divided by number of slits, as a function of magnitude. At bright magnitudes, most of the successfully extracted spectra have $Q=4$. Right panels: same as the left panels, only as a function of $(V-I)$ color.

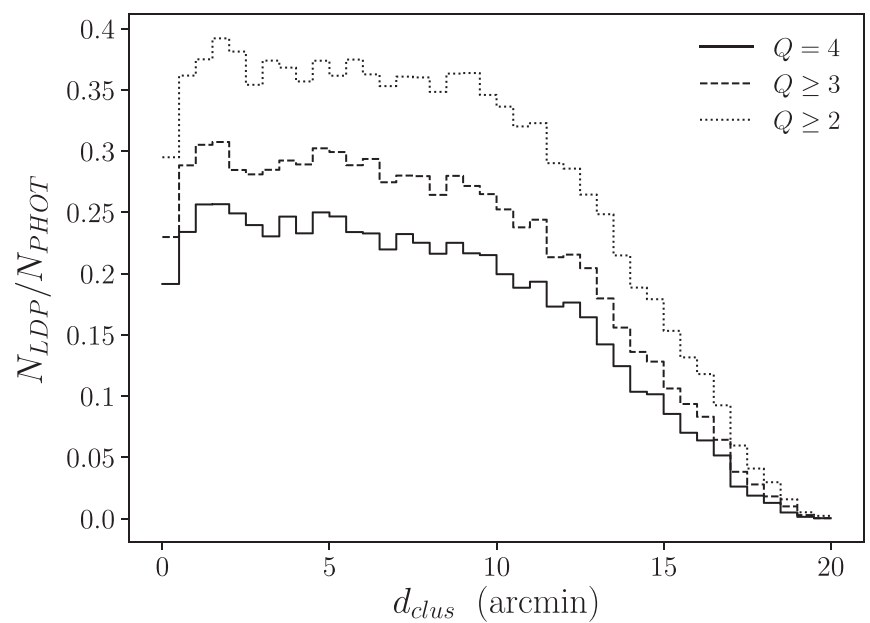

Figure 7. Fraction of photometric sources with LDP redshifts $\left(N_{\mathrm{LDP}} / N_{\mathrm{PHOT}}\right)$ as a function of clustercentric angular distance $\left(d_{\text {clus }}\right)$. A distance of $10^{\prime}$ corresponds to $\sim 4 \mathrm{Mpc}$, at $z=0.6$. Bin sizes are 0.5 , and solid, dashed, and dotted lines include redshifts with $Q=4, Q \geqslant 3$, and $Q \geqslant 2$, respectively.

is only $\approx 40 \%$ of that value. This affects our ability to find cluster galaxies within the cluster core, where the galaxy surface densities are higher (see Section 5).

\section{Cluster Infall Regions}

We use the theory of secondary infall (Fillmore \& Goldreich 1984; Bertschinger 1985; White \& Zaritsky 1992) to estimate (1) the masses that our clusters are expected to accrete by $z=0\left(M_{\text {infall }}\right)$ and (2) the projected radii at the cluster redshifts that encloses $M_{\text {infall }}\left(R_{\text {infall }}\right)$. Using the latter with the LDP data, we calculate the number of galaxies in the infall region $\left(N_{\text {infall }}\right)$. In Section 5.1, we calculate the expected evolution of the clusters in terms of mass and compare to semianalytic models. In Section 5.2, we focus on the dependence of $N_{\text {infall }}$ on cluster velocity dispersion and quantify its scatter. In Section 5.3, we examine the quiescent fraction of galaxies in different environments, using the red sequence galaxy fraction as a proxy, and quantify the amount of clustering in the infall regions.

\subsection{The Secondary Infall Model}

While previous studies of cluster infall regions have used the caustic technique (e.g., Geller et al. 1999; Rines et al. 2003; Rines \& Diaferio 2006; Serra et al. 2011; Owers et al. 2017), which identifies curves in galaxy position-radial velocity phase space that encompass those galaxies that are gravitationally bound to the cluster, such an analysis requires redshift accuracy greater than that of the LDP. Alternatively, we identify the infall regions of our clusters using the theory of secondary infall to define projected radii that encompass the infall region.

The secondary infall model describes how shells of mass centered on a cosmic perturbation evolve over time. The shells begin by expanding outward, until a time $t_{\text {turn }}$ when they turn around owing to the pull of gravity. The shells do not cross during this time, and there is a critical mass, $M_{*}$, enclosed by the shell that is marginally bound. All shells enclosing a mass less than $M_{*}$ eventually turn around at different times and 
Table 5

Mass Infall Model Results

\begin{tabular}{|c|c|c|c|c|c|c|c|c|}
\hline $\begin{array}{l}\text { Field } \\
\text { (1) }\end{array}$ & $\begin{array}{l}\text { Cluster } \\
\text { (2) }\end{array}$ & $\begin{array}{c}z \\
(3)\end{array}$ & $\begin{array}{c}R_{\text {infall }} \\
\text { (4) }\end{array}$ & $\begin{array}{l}\frac{R_{\text {infall }}}{R_{200}} \\
(5)\end{array}$ & $\begin{array}{c}M_{200, z}=0 \\
(6)\end{array}$ & $\begin{array}{c}\frac{M_{200, z=0}}{M_{200}} \\
\text { (7) }\end{array}$ & $\begin{array}{c}\sigma_{z}=0 \\
(8)\end{array}$ & $\begin{array}{c}\frac{\sigma_{z=0}}{\sigma} \\
(9)\end{array}$ \\
\hline 1 & Cl1018.8-1211 & 0.4734 & 3.06 & 3.28 & $2.01(14)$ & 1.32 & 533 & 1.10 \\
\hline 2 & C11037.9-1243 & 0.5783 & 2.07 & 3.60 & $5.67(13)$ & 1.40 & 357 & 1.12 \\
\hline 3 & C11037.9-1243a & 0.4252 & 3.31 & 3.12 & $2.72(14)$ & 1.28 & 584 & 1.09 \\
\hline 4 & Cl1040.7-1155 & 0.7043 & 2.75 & 3.94 & $1.27(14)$ & 1.50 & 478 & 1.14 \\
\hline 5 & Cl1054.4-1146 & 0.6972 & 3.87 & 3.92 & $3.55(14)$ & 1.49 & 673 & 1.14 \\
\hline 6 & C11054.7-1245 & 0.7498 & 3.32 & 4.05 & $2.21(14)$ & 1.53 & 581 & 1.15 \\
\hline 7 & Cl1059.2-1253 & 0.4564 & 3.19 & 3.23 & $2.33(14)$ & 1.31 & 558 & 1.09 \\
\hline 8 & Cl1103.7-1245a & 0.6261 & 2.20 & 3.73 & $6.61(13)$ & 1.43 & 379 & 1.13 \\
\hline 9 & Cl1103.7-1245b & 0.7031 & 1.66 & 3.93 & $2.78(13)$ & 1.49 & 288 & 1.14 \\
\hline 10 & Cl1138.2-1133 & 0.4796 & 4.62 & 3.30 & $6.88(14)$ & 1.32 & 804 & 1.10 \\
\hline 11 & Cl1216.8-1201 & 0.7943 & 6.70 & 4.15 & $1.82(15)$ & 1.57 & 1183 & 1.16 \\
\hline 12 & Cl1227.9-1138 & 0.6357 & 3.76 & 3.76 & $3.29(14)$ & 1.44 & 648 & 1.13 \\
\hline 13 & Cl1227.9-1138a & 0.5826 & 2.21 & 3.61 & $6.93(13)$ & 1.40 & 382 & 1.12 \\
\hline 14 & Cl1232.5-1250 & 0.5414 & 6.95 & 3.49 & $2.21(15)$ & 1.37 & 1199 & 1.11 \\
\hline 15 & Cl1301.7-1139 & 0.4828 & 4.34 & 3.31 & $5.69(14)$ & 1.33 & 755 & 1.10 \\
\hline 16 & Cl1301.7-1139a & 0.3969 & 2.37 & 3.02 & $1.05(14)$ & 1.26 & 423 & 1.08 \\
\hline 17 & C11353.0-1137 & 0.5882 & 4.33 & 3.63 & $5.16(14)$ & 1.41 & 746 & 1.12 \\
\hline 18 & Cl1354.2-1230 & 0.7620 & 4.27 & 4.08 & $4.70(14)$ & 1.54 & 749 & 1.16 \\
\hline 19 & Cl1354.2-1230a & 0.5952 & 2.82 & 3.65 & $1.42(14)$ & 1.41 & 486 & 1.12 \\
\hline 20 & C11411.1-1148 & 0.5195 & 4.54 & 3.43 & $6.27(14)$ & 1.35 & 785 & 1.11 \\
\hline 21 & Cl1420.3-1236 & 0.4962 & 1.38 & 3.35 & $1.82(13)$ & 1.34 & 240 & 1.10 \\
\hline
\end{tabular}

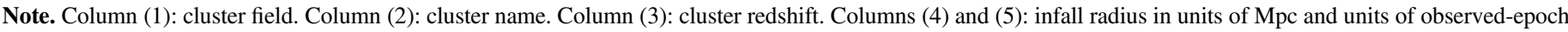

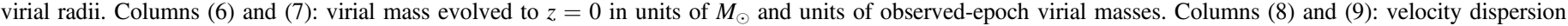
evolved to $z=0$ in units of $\mathrm{km} \mathrm{s}^{-1}$ and units of observed-epoch $\sigma$.

collapse, while shells at larger radii continue to expand forever. We follow the equations of White \& Zaritsky (1992), who assume an open universe with $\Omega_{\Lambda}=0$. Keeping $\Omega_{\Lambda}=0$ when calculating $M_{\text {infall }}$ and $R_{\text {infall }}$ does not significantly affect the analysis given the physical scales involved (e.g., Del Popolo et al. 2013), as we also confirm with our own comparison to cosmological simulations discussed below. For determining global quantities (e.g., connecting a time with a redshift and age), we continue to use $\Omega_{\Lambda}=0.7$.

Our aim is to compare the "mass" of the cluster at the observed time to that at the current time. We approximate the cluster's mass by measuring the mass of material that has reached the cluster center at least once by the time of interest. For the case of the mass at the observed time, that time corresponds to the age of the universe at the observed redshift. To calculate the mass that has reached the center, we use the equations of secondary infall and calculate the mass enclosed within the turnaround radius $\left(R_{\text {turn }}\right)$ at half the age of interest. For the observed clusters we approximate the enclosed mass using $M_{200}$ (Table 1) and then calculate $M_{*}$ in the following equation when we set the turnaround time $t_{\text {turn }}$ to half the age of the universe:

$$
t_{\text {turn }}\left(M_{\mathrm{enc}}\right)=\frac{\pi}{2} \frac{\Omega}{H(1-\Omega)^{3 / 2}}\left[\left(\frac{M_{*}}{M_{\mathrm{enc}}}\right)^{2 / 3}-1\right]^{-3 / 2} .
$$

Once $M_{*}$ is determined, we use Equation (4) again, with $t_{\text {turn }}$ equal to half the present age of the universe, to determine the enclosed mass or approximate $M_{200}$ at $z=0$. The ratio of the mass at the current time to that at the observed time is presented in Table 5. These quantities are calculated separately for each cluster, which is why two clusters with nearly the same velocity dispersion (e.g., clusters 3 and 6 in Table 5) can have quite different mass ratios. $R_{\text {turn }}$ for this shell is related to $t_{\text {turn }}$ through the simple equation for a free-falling test particle,

$$
t_{\text {turn }}\left(M_{200, z=0}\right)=\frac{\pi}{2} \sqrt{\frac{R_{\text {turn }}^{3}}{2 G M_{200, z=0}}} .
$$

While we solve for $R_{\text {turn }}$ for each cluster, which turns around when the universe is half its present age, what we are truly interested in is the location of these shells at $z=z_{\text {clus }}$. Therefore, we use the equation of motion for a uniform mass shell to evolve $R_{\text {turn }}$ to $z=z_{\text {clus }}$. These radial distances are the infall radii $\left(R_{\text {infall }}\right)$, the outer boundaries of the relevant infall regions for $z=0$ observations.

In defining radial distances, we center on the location of the BCG. However, the BCG may be offset from the distribution of mass, which would affect the definition of the infall region. We estimate the magnitude of these offsets from Figure 6 of White et al. (2005), which marks the BCG position relative to adaptively smoothed contours of cluster galaxy surface density. The offsets are $\lesssim 10 \%$ of $R_{\text {infall }}$ for all clusters except Cl1037 -1243 , whose BCG is offset by $\sim 25 \%$ of $R_{\text {infall }}$. These values are larger than the typical offsets found at $z \approx 0.5$ by Zitrin et al. (2012), but they find that the offsets are positively correlated with redshift and our clusters lie at higher $z$ than their sample. In addition, the galaxy distributions from White et al. (2005) include galaxies with photometric redshifts consistent with being close to the cluster redshift and therefore include a non-negligible number of interlopers that make the centering less precise. If we define the center to be the mean R.A. and decl. of the VLT/FORS2 spectroscopic sources, then 41 galaxies (12\% of the infalling population) are either removed or added by the new definition. However, the total number of infalling galaxies changes by less than $2 \%$ (because some are 
added, while some are removed from the infall region), and the fraction of red galaxies (Section 5.3) changes by only $0.4 \%$. As a final check, we randomly apply offsets of $\sim 0.2 \mathrm{Mpc}$ in various directions from the BCGs and find that similar numbers of galaxies are affected by the redefined infall regions. We conclude that reasonable uncertainties in the centering of the clusters do not strongly impact our results or conclusions.

In Table 5, we present the results of the models for our clusters. In addition to $R_{\text {infall }}$, we calculate the predicted mass (and corresponding velocity dispersion) at $z=0$ for our sample. The infall radii range from 1.2 to $6.7 \mathrm{Mpc}$; the ratio of $R_{\text {infall }}$ to $R_{200}$ is set entirely by the redshift of the cluster, in that higher- $z$ clusters have larger ratios, and range from $3.0 R_{200}$ to $4.2 R_{200}$. These are smaller than the "turnaround radii" calculated in other studies of cluster infall regions, such as those found in Rines \& Diaferio $\left(2006, \approx 4.75 R_{200}\right)$. However, their definition of infall region includes all galaxies that, with a velocity less than the cluster escape velocity, will eventually become incorporated into the cluster given enough time, while our model only includes galaxies that could have reached the center of the cluster by $z=0$.

We compare our infall radii to models using the Millennium Simulation (Springel et al. 2005), estimating the infall radii in the simulation using the fraction of galaxies at a given clustercentric distance that come to lie within $R_{200}$ at $z=0$. We considered $174 \sim 10^{14} M_{\odot}$ halos at $z \sim 0.6$, which have $R_{\text {infall }} \approx 3.5 R_{200}$ according to our analytic modeling. We find that $67 \% \pm 7 \%$ of the galaxies within $3.5 R_{200}$ at $z=0.62$ ultimately lie within the virial radius of the descendant halo (the errors are the 15th-85th percentiles). Because we assume that $100 \%$ of galaxies within $R_{\text {infall }}$ become "cluster galaxies" of the descendant halo, our prediction is good to $\approx 33 \%$. The difference arises from a variety of effects, including the assumption of spherical symmetry, the definition of an infalling galaxy as one that reaches $R=0$ at $z=0$, cluster galaxy dynamics such as merging and tidal stripping, and the presence of "backsplash" galaxies, which pass through the cluster core and then continue out to radii larger than $R_{200}$ (Balogh et al. 2000). We also acknowledge that galaxies from $R>R_{\text {infall }}$ may be measured within the virial radius at $z=0$, but we expect that this effect is small and subdominant to the other sources of uncertainty.

Our models predict an increase in cluster mass of $26 \%-57 \%$ from the observed epoch to the current one (Column (7) of Table 5). Velocity dispersion increases as

$$
\sigma \propto M^{1 / 3}\left[\Omega_{\Lambda}+\Omega_{0}(1+z)^{3}\right]^{1 / 6}
$$

which means that the mean growth corresponds to $\sigma$ increasing by $\approx 15 \%-25 \%$. The predicted $\sigma$ at $z=0$ agrees to within $\sim 10 \%$ of the predictions at a given mass that Poggianti et al. (2006) computed for $z=0.6$ clusters by combining the highresolution $N$-body simulations of Wechsler et al. (2002) with cluster concentration parameters from Bullock et al. (2001).

\subsection{Number of Infalling Galaxies}

We estimate the richness of the infall regions $\left(R_{200}<R<R_{\text {infall }}\right)$, which we define as the number of cluster galaxies in the infall region $\left(N_{\text {infall }}\right)$ above an absolute $B$-band magnitude of $M_{B}=$ -18.9 (corresponding to $R_{\mathrm{AUTO}} \approx 22.9$ for our highest-redshift cluster at $z=0.79$ ).

Because we do not have redshifts for every galaxy above this magnitude limit, to estimate $N_{\text {infall }}$, we use the number of

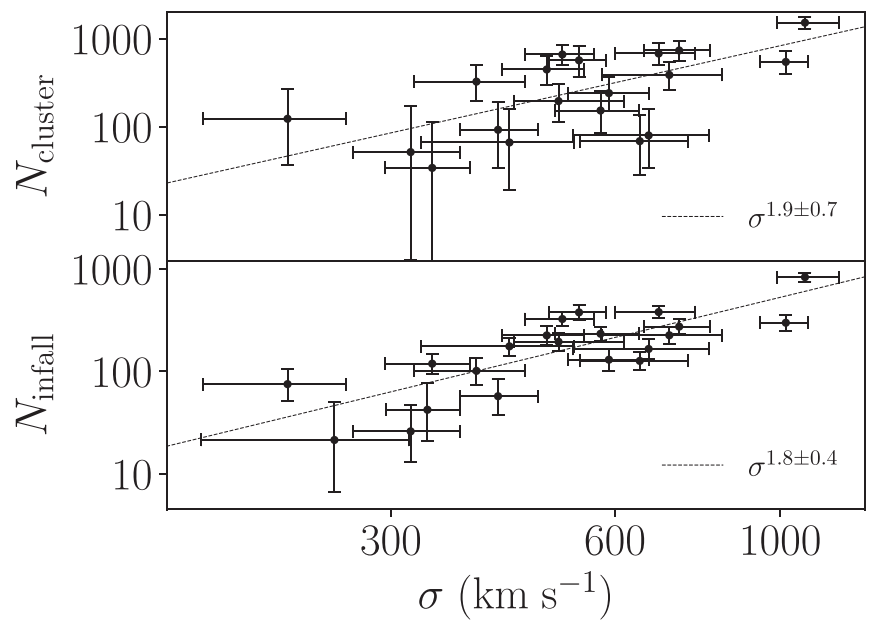

Figure 8. Top panel: number of galaxies within the virial radius $\left(N_{\text {cluster }}\right)$ as a function of cluster velocity dispersion $(\sigma)$. The dashed line is the best fit to the data. Bottom panel: same as the top panel, only for galaxies in the infall region $\left(N_{\text {infall }}\right)$.

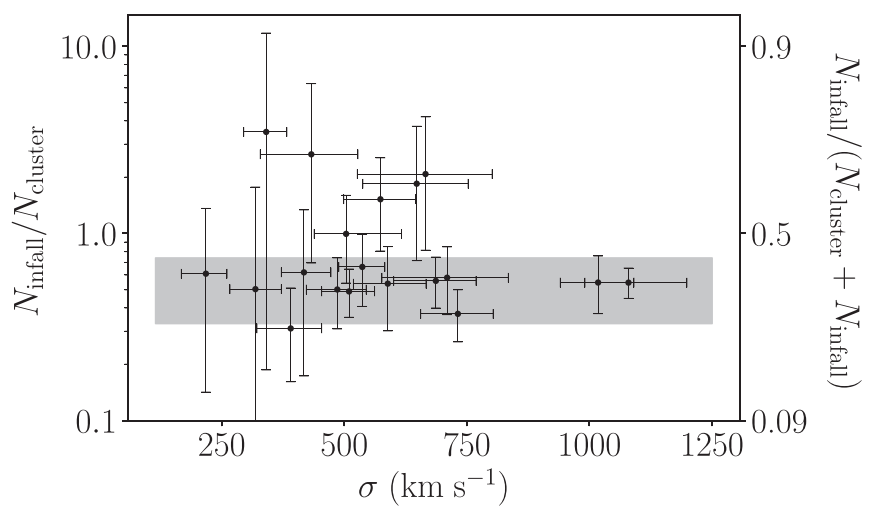

Figure 9. Ratio of infalling galaxies to cluster galaxies as a function of velocity dispersion $(\sigma)$. The horizontal gray bar comes from converting the expected mass increase based on the secondary infall models (Table 5) to galaxy number using $\sigma_{8}$ (see text). No trend is present, such that clusters over this range of $\sigma$ accrete proportionally similar numbers of galaxies as they evolve to $z=0$ (although they typically range from $\sim 0.3$ to 2.0 ).

photometric sources in the radial range $R_{200}<R<R_{\text {infall }}$, $N_{\text {phot }}$, multiplied by an estimate of what fraction of $N_{\text {phot }}$ lies within $\Delta z \pm 0.02$ of the cluster. We determine this fraction from the ratio of LDP-selected cluster members, $N_{\text {memb }}$, to the number of LDP slits, $N_{\text {slits }}$, limiting both to the infall region. This procedure accounts for the incomplete spatial sampling due to chip gaps and masked bright stars. We estimate the contamination from field galaxies $\left(f_{\text {contam }}\right)$ by using the fraction of field galaxies at that cluster redshift; these galaxies lie at $z_{\mathrm{clus}}$ but are observed in fields other than that particular cluster (excluding any that lie within another EDisCS cluster; see Section 5.3). Therefore, $N_{\text {infall }}$ is calculated as

$$
N_{\text {infall }}=N_{\text {phot }} \frac{N_{\text {memb }}}{N_{\text {slits }}}\left(1-f_{\text {contam }}\right) \text {. }
$$

We also estimate the number of cluster galaxies within $R_{200}$ at $z=z_{\text {clus }}, N_{\text {cluster, }}$ using the same methodology and magnitude limits, but apply an additional correction to account for close pairs that the LDP might miss (see Section 4.2.3). To make this correction, we can look at how many of the core cluster galaxies targeted with VLT/FORS2 were also targeted 
with the LDP and divide by that fraction, which is $18 \%$. This correction is slightly underestimated because the slit geometry resulted in a slight undersampling of close pairs within the EDisCS clusters. However, after applying this correction, our derived values of $N_{\text {cluster }}$ agree within the uncertainties to previous estimates made using spectroscopically confirmed EDisCS galaxies (Poggianti et al. 2010).

In Figure 8, we present a plot of $N_{\text {cluster }}$ and $N_{\text {infall }}$ versus $\sigma$. Errors on $N_{\text {cluster }}$ and $N_{\text {infall }}$ are Poissonian (calculated using the equations of Gehrels 1986), and errors in $\sigma$ come from Halliday et al. (2004) and Milvang-Jensen et al. (2008). We perform a linear regression that follows a Bayesian approach and accounts for errors in both $\sigma$ and $N$, using the IDL routine LINMIX_ERR.PRO written by Kelly (2007). We find $N_{\text {cluster }} \propto \sigma^{1.9 \pm 0.7}$ and $N_{\text {infall }} \propto \sigma^{1.8 \pm 0.4}$. While the trend for $N_{\text {cluster }}$ has a steeper dependence on $\sigma$ than that of the $z \sim 0$ clusters observed by Finn et al. (2008), who found $N_{\text {cluster }} \propto \sigma^{1.4}$, the results are within the uncertainties. Given the large errors, we find no statistically significant difference in the intercepts in either region. While more precise measurements are needed to reduce the uncertainty, our best-fit scalings between $N_{\text {cluster }}$ and $N_{\text {infall }}$ with $\sigma$ are comparable.

Given the similar slopes, we now examine whether clusters of greater mass accrete proportionally more or fewer galaxies over this redshift interval. Such behavior would have ramifications for the $\sigma$-dependent increase in S0s as a fraction of cluster galaxies (Just et al. 2010). For example, if more massive systems accrete a larger percentage of their galaxies at late times relative to less massive systems, then it could be that the (proportionally larger) infalling population diluted any increase in the S0 fraction in these systems, rather than that the less massive systems are intrinsically more efficient at converting spirals to S0s. In Figure 9, we compare the ratio of $N_{\text {infall }}$ to $N_{\text {cluster }}$ as a function of $\sigma$. We find that the relative size of the infalling population does not scale with cluster velocity dispersion. However, there is a considerable range in the ratio, from $\approx 15 \%$ to $300 \%$, with typical values between $\sim 30 \%$ and $200 \%$. To determine whether the scatter of $N_{\text {infall }}$ or $N_{\text {cluster }}$ dominates the scatter in the ratio, we set either the scatter in $N_{\text {infall }}$ or $N_{\text {cluster }}$ to zero and evaluated the scatter in the ratio. We found that setting the scatter in $N_{\text {infall }}$ to zero had minimal effect on the scatter in the ratio and conclude that it is the scatter in $N_{\text {cluster }}$ that dominates. This result highlights the importance of accounting for the large variation in cluster properties.

We now use $N_{\text {cluster }}$ and $N_{\text {infall }}$ to predict the mass evolution of our clusters based on the LDP data. We model the correspondence between our clusters at their observed epoch and at $z=0$ using the model described in Poggianti et al. (2006). In this model, we account for the enhanced clustering of galaxies relative to the underlying mass distribution, parameterized by $\sigma_{8}$, the rms fluctuation of galaxies in an $8 h^{-1} \mathrm{Mpc}$ sphere relative to fluctuation in mass, and adopt a recent value, $\sigma_{8}=0.81$ (Jarosik et al. 2011).

From Table 5, we find that the typical increase in mass predicted by our adopted secondary infall models is $26 \%-57 \%$, or in terms of galaxy number, $33 \%-74 \%$. This is shown as a gray band in Figure 9, where it is consistent with our measured values of $N_{\text {infall }} / N_{\text {cluster, }}$, which are typically $\sim 30 \%-150 \%$ (24\%-110\% in mass). These values are lower than the factor of two mass increase predicted for $0.1<z<0.3$ clusters of Rines et al. (2013), although their use of the caustic technique means that their prediction should be higher than ours, because theirs is for the final cluster mass in the distant future, not $z=0$. Similarly, Dressler et al. (2013) find that among very rich clusters at $z \sim 0.4$, the number of galaxies in infalling groups will roughly double the mass of the clusters by the present, which is larger than our estimate but still consistent within the scatter. That we find a comparable mass increase among some of our clusters that have lower velocity dispersions than their sample $\left(\approx 800-1100 \mathrm{~km} \mathrm{~s}^{-1}\right)$ further supports our conclusion from Figure 9 that more massive systems do not accrete proportionally more galaxies as they evolve.

Our cluster sample spans redshifts between $z=0.39$ and $z=0.79$, corresponding to galaxy infall times of $4.3-6.8 \mathrm{Gyr}$. We have investigated the impact that this range of infall times has on the ratio $N_{\text {infall }} / N_{\text {cluster }}$. We fit a power law in redshift to the ratio and find a best-fit index of 1.34. After subtracting off the redshift dependence, we find no significant difference in the mean or the scatter of the cluster ratios. We conclude that even with our wide range of infall times, our results are consistent with the secondary infall model.

\subsection{Optical Properties of the Cluster and Infalling Galaxies}

To estimate the quiescent fraction of galaxies in different environments, we use the fraction of optically red galaxies. We examine the CMDs of our clusters and compare the red fractions of core, infalling, and field galaxies. To construct field samples for each cluster, we select galaxies at the same redshift but observed in fields other than the cluster's (excluding any that overlap in redshift with the EDisCS cluster of that particular field). Note that (1) this means that there are significantly more galaxies in a given field sample than in the corresponding cluster, because they are drawn from multiple fields, and (2) we combine a subset of these field samples in some of the analyses below, so we distinguish between "individual field samples" and a "combined field sample," the reason and details for which are described below.

For our CMDs, we use rest-frame $U-B$ colors and absolute $B$-band magnitudes calculated using EAZY (Brammer et al. 2008). In Figure 10 we present observed-frame CMDs for the 21 clusters. We measure the color-magnitude relations (CMRs) for all of our clusters by assuming zero slope and fitting the WFI $U-B$ colors of the subset of cluster galaxies that have FORS2 spectra showing no [O II] emission. The CMRs measured this way are in agreement with the apparent red sequences of LDP-selected cluster galaxies (Figure 10). In what follows, we define galaxies with colors within 0.2 of the CMR or redder as red, while the remaining galaxies are classified as blue.

Because some studies have shown that environmentally driven galaxy evolution is correlated with the velocity dispersion of the group/cluster (e.g., Poggianti et al. 2009; Just et al. 2010), in Figure 11 we plot the red fraction of the cluster and infalling samples for each cluster as a function of $\sigma$. To compare to the field, we create a "combined field sample." The "individual field samples" in Figure 10 have galaxies common to more than one sample. To avoid this multiple counting in the "combined field sample," we select the "individual field samples" of eight clusters that span the full redshift range from 0.4 to 0.8 but do not overlap in redshift. The red fraction for the combined field sample is also shown in Figure 11. We do not find a correlation between red fraction and $\sigma$ in the cluster environment, consistent with the findings of 

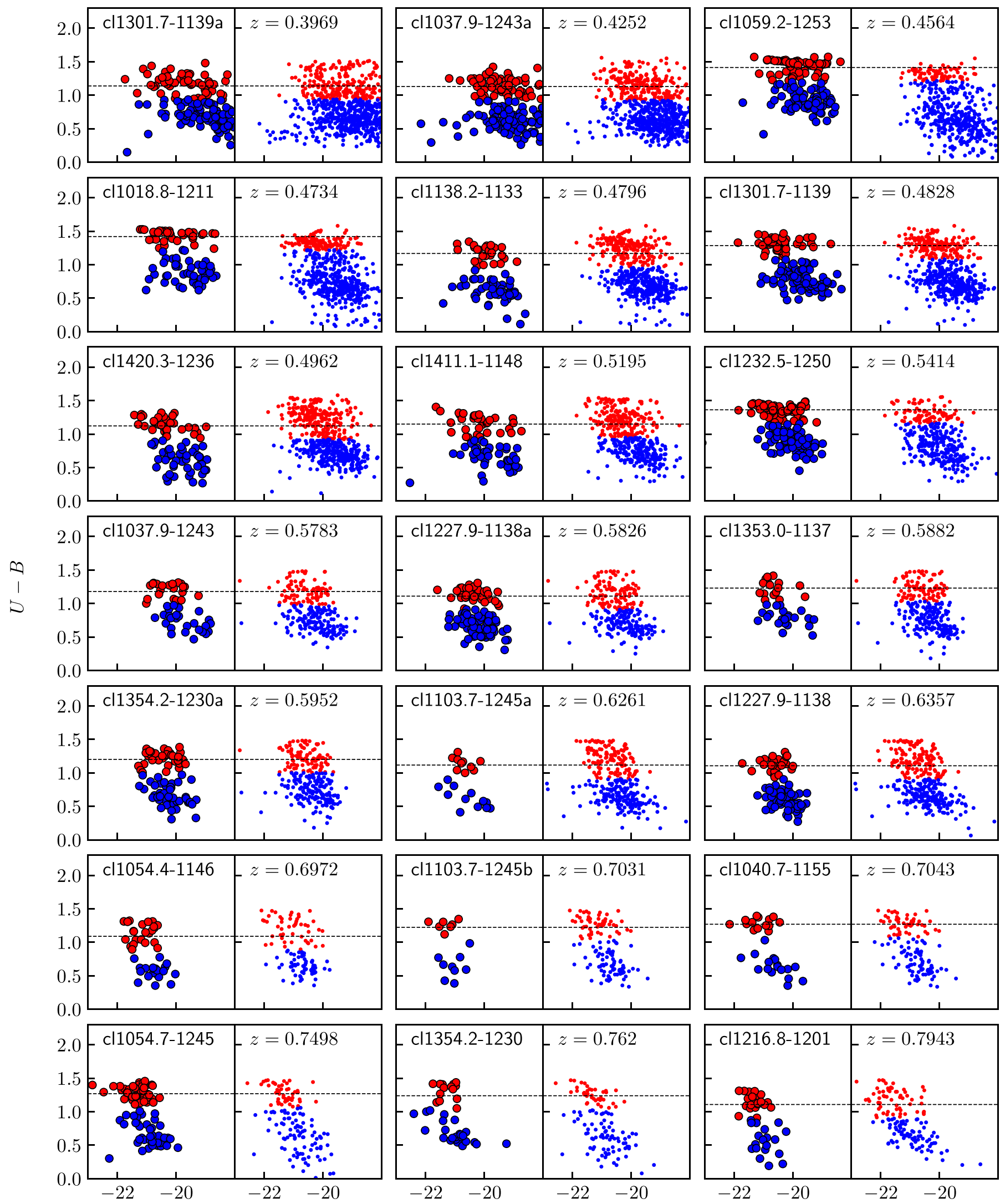

$M_{B}$

Figure 10. CMDs for our clusters in rest-frame $U-B$ vs. absolute $B$-band magnitude. The left frames show LDP-selected cluster members as circles that are colored based on our red/blue definition, while the right frames show the individual field samples. Dotted lines mark the cluster CMRs. Note that because of the way field samples are constructed, they contain many more galaxies than their corresponding cluster. 


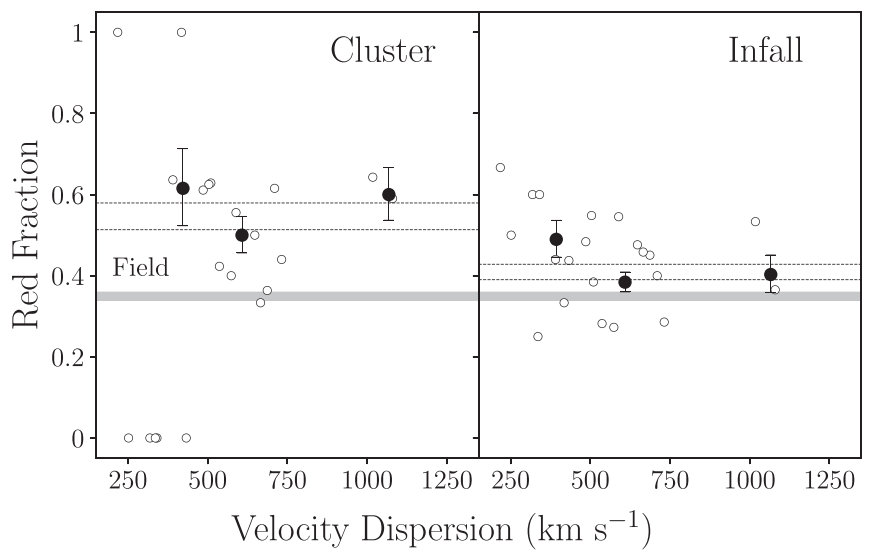

Figure 11. Red fraction as a function of cluster velocity dispersion for the cluster and infalling populations. Individual clusters are shown as open circles (with error bars suppressed for clarity), while large filled circles are the red fractions using all of the galaxies that lie in clusters with $\sigma<500 \mathrm{~km} \mathrm{~s}^{-1}, 500$ $\mathrm{km} \mathrm{s}^{-1}<\sigma<1000 \mathrm{~km} \mathrm{~s}^{-1}$, and $\sigma>1000 \mathrm{~km} \mathrm{~s}^{-1}$. Horizontal dotted lines show the mean \pm the rms of the overall red fraction for all clusters. Similarly, the overall red fraction of the combined field sample is shown as a shaded horizontal line, with a width corresponding to \pm the rms of the field red fraction. There are no statistically significant trends of red fraction with $\sigma$ in either environment. However, the red fraction decreases as one moves from the cores of clusters to the field.

Valentinuzzi et al. (2011) and Blanton \& Moustakas (2009), and find no correlation in the infalling sample either. However, the red fraction increases as one moves from the most isolated environment to the cores of clusters, from $36 \% \pm 1 \%$ in the field to $38 \% \pm 2 \%$ in the infall region and $55 \% \pm 3 \%$ in the virial region.

The red fraction in the infall regions is slightly elevated relative to the field for the lowest-mass clusters, but overall the sample is consistent with the field. Within the virial radius, the red fraction is elevated to $4.3 \sigma$ above the infall region and $5.5 \sigma$ above the field. Because galaxies move $\sim$ Mpc distances over $\sim$ Gyr timescales, the quenching of star formation could begin to occur in the infall region (e.g., Balogh et al. 2000), or even primarily occur there, with the higher red fraction within $R_{200}$ owing to the lag between the start of quenching and the time for its effects to become apparent. With high-resolution imaging, one would be able to assess whether the infalling red galaxies exhibit early-type morphologies or perhaps a transitory phase as passive disks.

Rudnick et al. (2009) find that the total light on the red sequence for 16 of the EDisCS clusters must increase by a factor of $\sim 1-3$ by $z=0$. We predict that the clusters in our sample will grow by a factor of $\sim 2.1$ in number of galaxies (Figure 9). Given that the red fractions within the cluster and infall regions are $55 \%$ and $38 \%$, respectively, passive galaxies already identified as such in the infall regions will increase the $z=0$ total red sequence light by a factor of $\sim 1.8$. We conclude that significant further quenching of blue galaxies in the infall regions as the clusters evolve to $z=0$ is not required by our sample. Although the uncertainties remain large given the limitations of the current sample and we cannot exclude significant further quenching, this line of reasoning holds promise as a consistency check on quenching models.

"Preprocessing" has been suggested as a way of transforming galaxies in locally overdense clumps prior to their incorporation

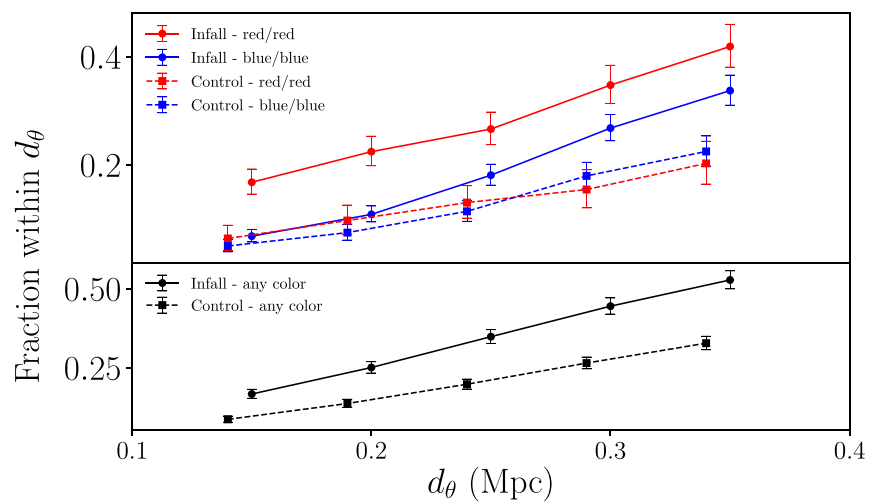

Figure 12. Top panel: fraction of infalling red and blue galaxies (red and blue circles, respectively) with at least one other infalling red/blue galaxy within a projected distance $d_{\theta}$. Also shown are the fractions for a control sample consisting of galaxies at the same redshifts and radial distances as the infall regions (red and blue squares). The control data are slightly offset in $d_{\theta}$ for clarity only. Bottom panel: similar to the top panel, only showing the fraction of infalling and control galaxies having a neighbor of either color within a distance $d_{\theta}$.

into the cluster (e.g., Zabludoff \& Mulchaey 1998; Moran et al. 2007; Kautsch et al. 2008; Dressler et al. 2013; Haines et al. 2013; Lopes et al. 2014; Cybulski et al. 2014). The elevated red fraction in the infall regions for low-mass clusters, where locally overdense clumps are expected to exist, is consistent with preprocessing. In the higher-mass clusters, a mixture of overdense quenching and underdense blue regions may result in an average red fraction that is barely elevated with respect to the field. However, this result does not rule out an additional global mechanism for quenching star formation, one that affects all galaxies at a given clustercentric radius equally. To explore this scenario further, we measure the amount of clustering among the galaxies in the infall regions, which will provide more direct evidence for the association of preprocessing with local overdensities. We measure the fraction of infalling red/blue galaxies with at least one infalling neighbor of similar color within a projected distance $d_{\theta}$, which we denote $F\left(<d_{\theta}\right)$, for values of $d_{\theta}$ ranging from 0.15 to $0.35 \mathrm{Mpc}$. A control sample is constructed where we select galaxies that (1) lie within the infall region of the cluster that they were imaged in; (2) are not a member of the cluster that they are imaged in, $z_{\mathrm{cl}}^{\text {imaged }}-z_{\mathrm{LDP}}>0.02$; and (3) have a redshift consistent with cluster member for another cluster in the sample, $z_{\mathrm{cl}}-z_{\mathrm{LDP}}<0.02$.

We present these distributions in Figure 12. The clearest result is the elevated fractions of red galaxies that have red neighbors within the infall region relative to either the control or the blue infall galaxies. A second notable finding is that the infalling blue galaxies are not significantly more clustered at small separations $\left(d_{\theta}<0.2 \mathrm{Mpc}\right)$ than the control blue galaxies. Alternatively, we measure the red fraction among close pairs of galaxies and compare to those without a neighbor (Figure 13). From both figures, we find that clustered galaxies are significantly more likely to be red than those without a neighbor, and this effect is more significant in the infall regions than in the control sample. Evidently, at these length scales the infall regions show signs of enhanced clustering of red galaxies, consistent with "preprocessing," in which local overdensities, rather than global environment, quench star formation prior to their incorporation into the cluster. 


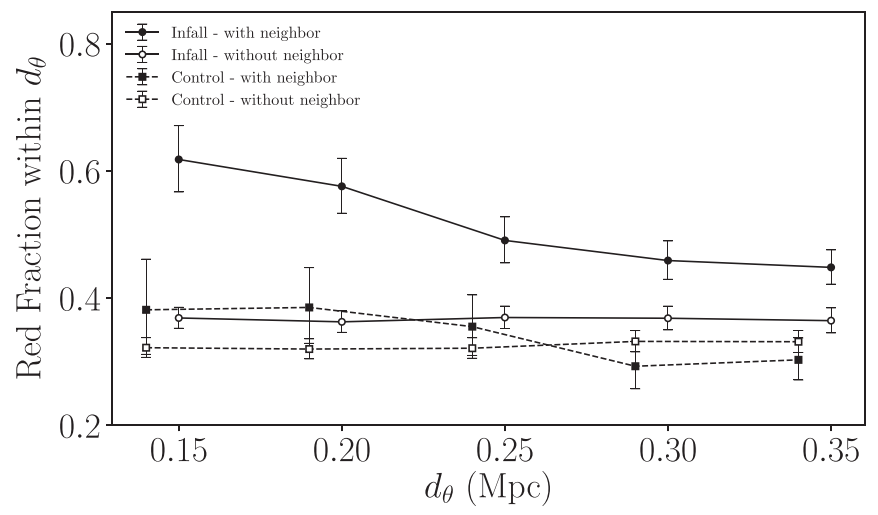

Figure 13. Red fraction of galaxies with or without a neighbor within a projected distance $d_{\theta}$, for both the infalling and control samples. Galaxies with close neighbors are more likely to be red in both samples, but in the infall region the enhancement in red fraction is higher than for the control sample.

We see the enhanced clustering and elevated red fraction among the infalling sample (Figures 12 and 13) relative to the control (i.e., field) sample. But despite our definition of clustering, based on having a neighbor within $d_{\theta}$, being the same for both samples, the red fractions are different. Therefore, either the infalling galaxies lie in higher local overdensities, or they experience an additional effect unrelated to local density. The spatial sampling rate of our LDP spectra means that we cannot directly compare the numbers of spectroscopically confirmed galaxies within $d_{\theta}$ between the two samples. Instead, we compare (1) the number of photometric sources and (2) the total $R$-band luminosity within $d_{\theta}$ to estimate the overdensities. We find that, relative to the control sample, the infalling cluster members have $\sim 3 \pm 2$ galaxies $\mathrm{Mpc}^{-2}$ more neighbors and contain $\sim 45 \% \pm 7 \%$ more $R$-band luminosity within $d_{\theta}$. While a more accurate measurement of local density using a higher sampling of redshifts would be preferable, this result is consistent with the idea that the infalling galaxies lie in higher local overdensities than the control galaxies, resulting in more clustering and a higher red fraction in the infalling sample (Figures 12 and 13).

The outlier rate for $Q=4$ galaxies is $12 \%$ (Section 4.2.1), which may have an impact on the results presented in this section as a result of contamination of the cluster samples by field galaxies. Given that we found no redshift or color dependence of the outlier rate, we expect both galaxies at the cluster redshift and field galaxies to be affected equally by redshift inaccuracy. We use the CMDs to estimate the relative numbers of cluster and field galaxies and find that the field galaxies constitute $\approx 30 \%$ of the total at a given redshift. Therefore, we expect a contamination of $12 \% \times 30 \%=3.6 \%$ field galaxies in the analyses above and do not expect this to be significant enough to alter our main results.

Dressler et al. (2013) found that quiescent and post-starburst (PSB) galaxies are preferentially found in denser environments, including infalling groups in the outskirts of rich clusters at $z \sim 0.4$. Furthermore, they identified a positive correlation between the fraction of quiescent and PSB galaxies in the infalling groups with increasing group mass, which they interpreted as evidence for "preprocessing." While we are unable to identify PSB galaxies with the LDP resolution, a prediction based on these results is that our infall regions contain a higher number of these galaxies than the field. If some fraction of our blue galaxies are PSB galaxies, then the quiescent plus PSB fraction in the infall regions would be even more different than the field value. Higher-resolution spectroscopy is needed to test this hypothesis.

In addition to environment, star formation is correlated with galaxy stellar mass (e.g., Kauffmann et al. 2003; Pasquali et al. 2009; Peng et al. 2010). Thomas et al. (2010) find that stellar mass may even be entirely responsible for the formation of early-type galaxies, at least at masses $\gtrsim 10^{11} M_{\odot}$. We explore whether the clustered and isolated galaxies (defined by a separation $d_{\theta}$ ) have different mass distributions. Following the prescription of Bell et al. (2003), we estimate stellar masses using $B$-band mass-to-light ratios, $\left(M_{*} / L\right)_{B}$, that are derived from $B-V$ rest-frame colors via

$$
\log \left(M_{*} / L\right)_{B}=1.737(B-V)-0.942,
$$

after converting our magnitudes to the Vega system. This assumes a diet Salpeter initial mass function as defined in Bell $\&$ de Jong (2001). Using $M_{B}=5.45$ for the Sun, a galaxy with $M_{B}=-19.5$ and $B-V=1$ has a stellar mass of $\log \left(M_{*} / M_{\odot}\right)=10.8$. Since our infalling galaxies span a mass range $\log \left(M_{*} / M_{\odot}\right)=9-12$, we repeat the analyses of Figures 12 and 13 but restricting to a narrower mass range, $\log \left(M_{*} / M_{\odot}\right)=10-11$, which is roughly symmetric about the median mass $\left(\log \left(M_{*} / M_{\odot}\right) \approx 10.5\right)$. For this narrower mass range, infalling red galaxies are significantly more likely to have a neighbor than blue galaxies, by $\approx 12 \% \pm 5 \%$ compared to $\approx 2 \% \pm 2 \%$ for the control sample. However, we cannot statistically conclude that infalling galaxies of either color are more likely to have a neighbor of the same color than the control sample, finding $\Delta F\left(<d_{\theta}\right) \approx 2 \% \pm 2 \%$. As when considering the full mass range, the infalling galaxies with a neighbor have a higher red fraction than isolated galaxies, by $\approx 50 \% \pm 12 \%$ compared to $\approx 10 \% \pm 4 \%$ for the control sample. An even narrower choice of masses than this leaves the results qualitatively unchanged, although the number of galaxies becomes too few to reach statistically significant conclusions like those listed above. While larger numbers of galaxies would help conclusively rule out a significant mass effect, based on these results we conclude that the enhanced red fraction among clustered galaxies is consistent with a primarily preprocessed origin.

Until now we have assumed that all galaxies in the infall regions are falling in for the first time. However, at these clustercentric radii there exists a population of galaxies that have already passed through the virial region in the past, socalled "backsplash" galaxies (Gill et al. 2005). These galaxies may have been quenched on their initial passage (or passages) through the main body of the cluster, independent of any preprocessing, and therefore must be accounted for. Balogh et al. (2000) suggest that as many as $54 \% \pm 20 \%$ of galaxies at distances between $R_{200}$ and $2 R_{200}$ are members of this backsplash population. Other studies involving backsplash galaxies focus on distances between the virial radius and 2.5 times the virial radius (e.g., Mamon et al. 2004; Oman et al. 2013). $N$-body simulations show that satellite galaxies ejected from the host halo could constitute $\sim 10 \%$ of galaxies at $2 R_{200}-5 R_{200}$ (Wetzel et al. 2014). While these studies show that 
a sizable fraction of backsplash galaxies could be present in the infall regions, these are not expected to be as clustered as firstinfall galaxies because we expect that their cluster crossing separated galaxies that fell in together. We base that expectation on the consideration that infalling groups are of roughly the same physical scale as the cluster core but of lower mass, and therefore we expect tidal effects to dissolve the group. The efficiency and ubiquity of this process need to be evaluated quantitatively with simulations. If our conjecture is correct, then a dominant contribution by backsplash galaxies is at odds with our findings in Figures 12 and 13, in which the red galaxies are significantly more clustered and the red fraction of clustered galaxies is significantly higher than those without close neighbors. However, we cannot quantify the significance of the backsplash population in driving the enhanced quenching we interpret in the infall regions. It will be quite difficult to disentangle these two populations.

Matches may also include some associations that are not physical but are rather chance projections. These could include both matches with unassociated galaxies that are cluster members and matches with galaxies beyond the cluster environment. Given our poor redshift resolution, there is no way to identify such cases using our data. Ultimately, a comprehensive analysis of simulated data in a manner that is consistent with our observing methodology should be carried out but is beyond the scope of this paper. The excess found relative to the control in the correlation of red galaxies with other red galaxies suggests that the bulk of the signal is real, given that at these radii the cluster environment is not dominated by red galaxies, but quantitative conclusions will await the full simulations. Given the large uncertainties in our understanding of how well infalling structures survive, any detailed analysis of the data may be premature.

\section{Conclusion}

We present a spectroscopic survey of 21 EDisCS clusters at $0.4<z<0.8$ using LDP/IMACS low-resolution spectroscopy. This survey contains 35,280 galaxies (with 1763 within \pm 0.02 of the corresponding cluster redshift) and has an accuracy of $\sigma_{z}=0.007$.

We have isolated the galaxies in the infall regions of these clusters using the LDP data and a simple model of secondary infall. The projected distance that encompasses the infalling galaxy population, $R_{\text {infall }}$, agrees to simulations within $\sim 30 \%$. The predicted cluster velocity dispersions at $z=0$ agree with the models of Poggianti et al. (2006) to $10 \%$.

With the LDP data, we identified the number of galaxies in the infall regions and estimate that $\sim 30 \%-70 \%$ of the $z=0$ cluster population lies outside the virial radius at $z \sim 0.6$, a result that is not sensitive to the mass of the cluster over the range of cluster mass investigated here. This result demonstrates that studying the infalling population is crucial to understanding how a significant portion of the galaxy population evolves. Furthermore, the ratio of the number of infalling galaxies to cluster galaxies is typically $\sim 0.3-1.5$. The full range of this ratio is $\approx 10 \%-300 \%$, highlighting the large cluster-to-cluster variation that exists.

The red fraction in the infall regions is intermediate to that in the field and clusters for low cluster masses. This suggests that the process of quenching star formation has begun outside of the virial radius, an effect previously measured at $z \sim 0$ (Lewis et al. 2002; Gómez et al. 2003). Furthermore, galaxies in the infall regions show enhanced clustering, and the more highly clustered galaxies also show an elevated red fraction. These trends are indicative of "preprocessing," in which galaxy star formation is shut off in local galaxy overdensities prior to the incorporation of the galaxies into the cluster, although backsplash galaxies may play a role. Our sample lies at $z \sim 0.6$, before the epoch at which significant numbers of S0s begin to populate the cores of clusters (Dressler et al. 1997; Fasano et al. 2000), so it is plausible that the S0s in those cores are the remnants of quenched infalling galaxies that we see clustered in the infall regions. Higher-resolution imaging is required to identify the morphologies of these possible progenitors.

This data set enables the direct study of galaxies in the infalling regions of moderate-mass clusters at intermediate redshifts. It clearly demonstrates that further studies seeking to understand the mechanisms that halt star formation in dense environments should target not just the virialized regions of clusters but the outskirts as well.

We thank an anonymous referee for insightful comments that have improved the content and presentation of this paper. D.W.J. and D.Z. acknowledge financial support from NASA LTSA award NNG05GE82G and GALEX grant NNX11AI47G. G.D.L. acknowledges financial support from the European Research Council under European Community's Seventh Framework Programme (FP7/2007-2013)/ERC grant agreement No. 202781. P.J. acknowledges support by the Swiss National Science Foundation (SNSF).

\section{Appendix Spatial Maps of Cluster Members}

In Figure 14, we present the spatial map of galaxies in and around our clusters. We mark cluster member galaxies that we classify as red or blue in their respective colors, and all other detected galaxies in the field as small black points. We also draw black circles corresponding to the virial radius (inner circle) and the infall radius (outer circle) for each of our clusters. 

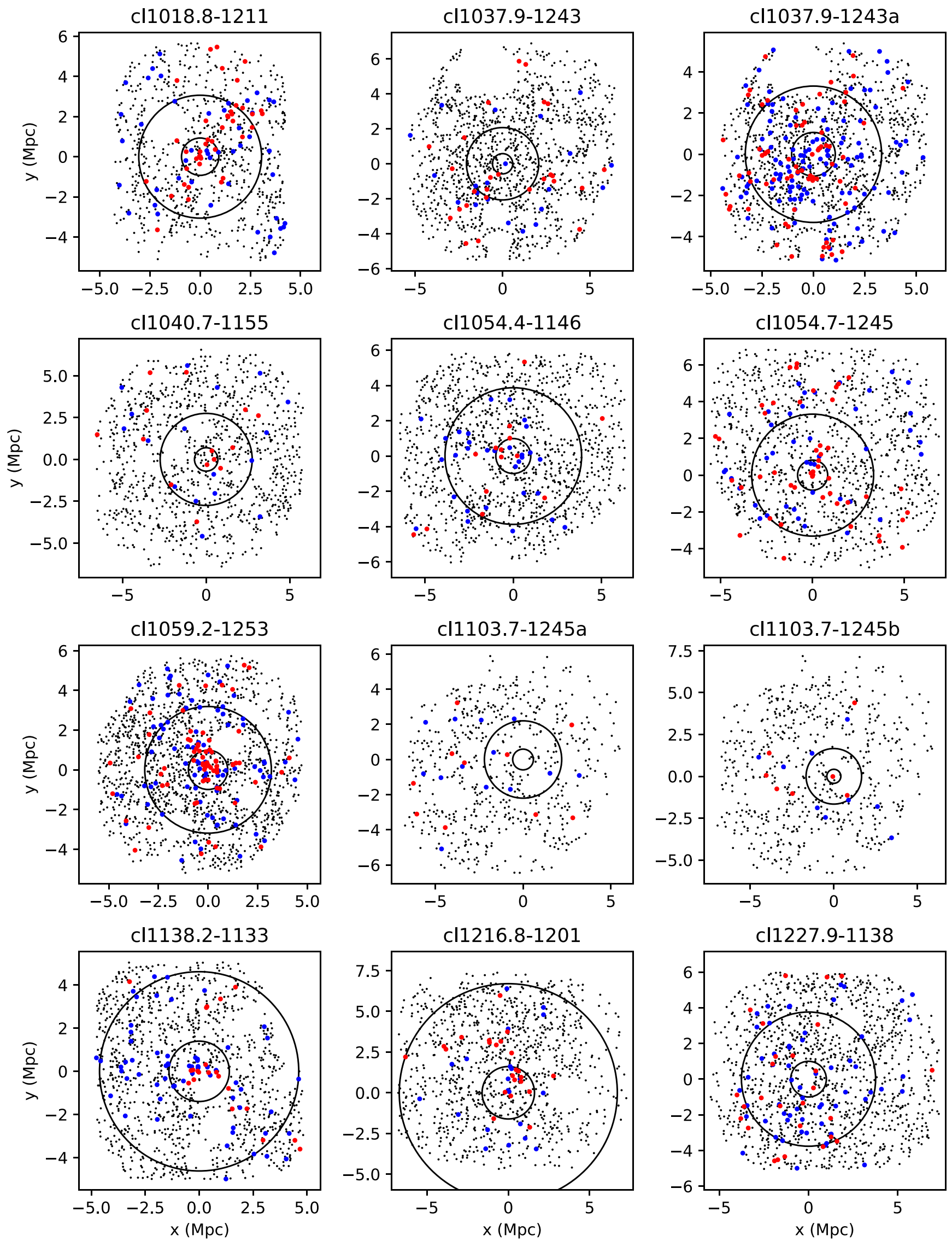

Figure 14. Spatial maps of our clusters. Red and blue circles mark cluster members meeting our red/blue definitions. The small black points represent all of the other detected galaxies in the fields. Black circles corresponding to the virial and infall radii are also shown. 

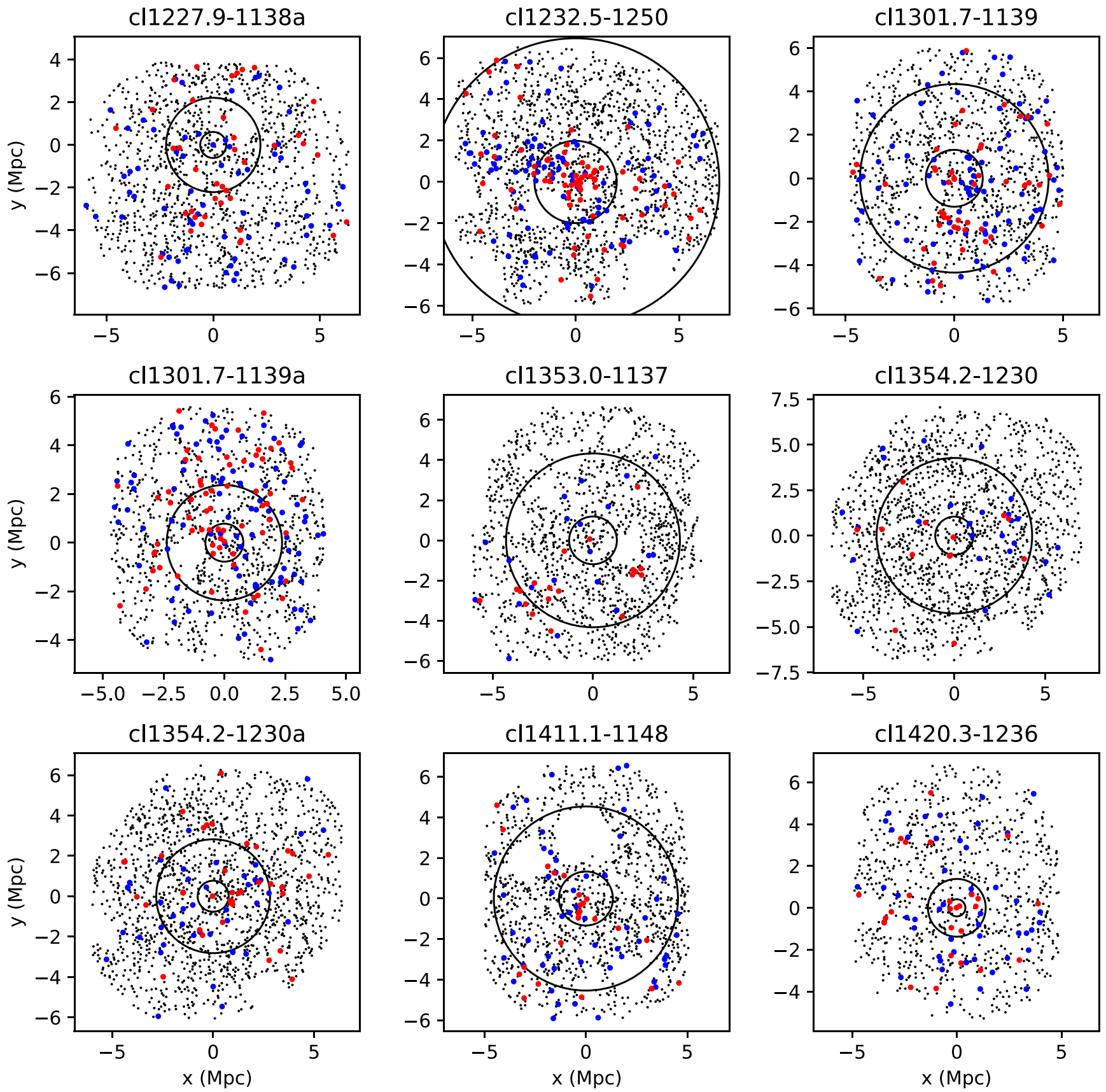

Figure 14. (Continued.)

\section{ORCID iDs}

Dennis W. Just (10 https://orcid.org/0000-0001-8580-630X Matthew Kirby (i) https://orcid.org/0000-0002-4788-200X Dennis Zaritsky (i) https://orcid.org/0000-0002-5177-727X Gregory Rudnick (1) https://orcid.org/0000-0001-5851-1856 Vandana Desai 10 https://orcid.org/0000-0002-1340-0543 Rose Finn (ㄷ) https://orcid.org/0000-0001-8518-4862 Bianca Poggianti (i) https://orcid.org/0000-0001-8751-8360

\section{References}

Baade, D., Meisenheimer, K., Iwert, O., et al. 1999, Msngr, 95, 15 Bahé, Y. M., McCarthy, I. G., Balogh, M. L., \& Font, A. S. 2013, MNRAS, 430, 3017

Balogh, M. L., Navarro, J. F., \& Morris, S. L. 2000, ApJ, 540, 113
Banse, K., Ponz, D., Ounnas, C., Grosbol, P., \& Warmels, R. 1988 , Instrumentation for Ground-Based Optical Astronomy, Present and Future. The Ninth Santa Cruz Summer Workshop in Astronomy and Astrophysics (New York: Springer-Verlag), 431

Bell, E. F., \& de Jong, R. S. 2001, ApJ, 550, 212

Bell, E. F., McIntosh, D. H., Katz, N., \& Weinberg, M. D. 2003, ApJS, 149, 289

Bertin, E. 2006, adass XV, 351, 112

Bertin, E., \& Arnouts, S. 1996, A\&AS, 117, 393

Bertin, E., Mellier, Y., Radovich, M., et al. 2002, adass XI, 281, 228 Bertschinger, E. 1985, ApJS, 58, 39

Bigelow, B. C., Dressler, A. M., Shectman, S. A., \& Epps, H. W. 1998, Proc. SPIE, 3355, 225

Blanton, M. R., \& Moustakas, J. 2009, ARA\&A, 47, 159

Blanton, M. R., \& Roweis, S. 2007, AJ, 133, 734

Bösch, B., Böhm, A., Wolf, C., et al. 2013, A\&A, 549, A142

Brammer, G. B., van Dokkum, P. G., \& Coppi, P. 2008, ApJ, 686, 1503

Bruzual, G., \& Charlot, S. 2003, MNRAS, 344, 1000 
Bullock, J. S., Kolatt, T. S., Sigad, Y., et al. 2001, MNRAS, 321, 559 Bundy, K., Scarlata, C., Carollo, C. M., et al. 2010, ApJ, 719, 1969 Cardelli, J. A., Clayton, G. C., \& Mathis, J. S. 1989, ApJ, 345, 245 Christlein, D., \& Zabludoff, A. I. 2004, ApJ, 616, 192

Christodoulou, L., Eminian, C., Loveday, J., et al. 2012, MNRAS, 425, 1527 Clowe, D., \& Schneider, P. 2001, A\&A, 379, 384

Clowe, D., \& Schneider, P. 2002, A\&A, 395, 385

Clowe, D., Schneider, P., Aragón-Salamanca, A., et al. 2006, A\&A, 451, 395

Coil, A. L., Blanton, M. R., Burles, S. M., et al. 2011, ApJ, 741, 8

Cool, R. J., Moustakas, J., Blanton, M. R., et al. 2013, ApJ, 767, 118

Cortese, L., Gavazzi, G., Boselli, A., et al. 2006, A\&A, 453, 847

Cybulski, R., Yun, M. S., Fazio, G. G., \& Gutermuth, R. A. 2014, MNRAS, 439, 3564

Del Popolo, A., Pace, F., \& Lima, J. A. S. 2013, IJMPD, 22, 1350038

Desai, V., Dalcanton, J. J., Aragón-Salamanca, A., et al. 2007, ApJ, 660, 1151

Dressler, A. 1980, ApJ, 236, 351

Dressler, A., Hare, T., Bigelow, B. C., \& Osip, D. J. 2006, Proc. SPIE, 6269, $62690 \mathrm{~F}$

Dressler, A., Oemler, A., Poggianti, B. M., et al. 2013, ApJ, 770, 62

Dressler, A., Oemler, A., Jr., Couch, W. J., et al. 1997, ApJ, 490, 577

Fasano, G., Poggianti, B. M., Couch, W. J., et al. 2000, ApJ, 542, 673

Fillmore, J. A., \& Goldreich, P. 1984, ApJ, 281, 1

Finn, R. A., Balogh, M. L., Zaritsky, D., Miller, C. J., \& Nichol, R. C. 2008, ApJ, 679, 279

Finn, R. A., Desai, V., Rudnick, G., et al. 2010, ApJ, 720, 87

Finn, R. A., Zaritsky, D., McCarthy, D. W., Jr., et al. 2005, ApJ, 630, 206

Gehrels, N. 1986, ApJ, 303, 336

Geller, M. J., Diaferio, A., \& Kurtz, M. J. 1999, ApJL, 517, L23

Gill, S. P. D., Knebe, A., \& Gibson, B. K. 2005, MNRAS, 356, 1327

Gómez, P. L., Nichol, R. C., Miller, C. J., et al. 2003, ApJ, 584, 210

Gonzalez, A. H., Zaritsky, D., Dalcanton, J. J., \& Nelson, A. 2001, ApJS, 137,117

Guennou, L., Adami, C., Ulmer, M. P., et al. 2010, A\&A, 523, A21

Haines, C. P., Pereira, M. J., Smith, G. P., et al. 2013, ApJ, 775, 126

Halliday, C., Milvang-Jensen, B., Poirier, S., et al. 2004, A\&A, 427, 397

Hauschildt, P. H., Allard, F., \& Baron, E. 1999, ApJ, 512, 377

Hogg, D. W., Blanton, M. R., Brinchmann, J., et al. 2004, ApJL, 601, L29

Jarosik, N., Bennett, C. L., Dunkley, J., et al. 2011, ApJS, 192, 14

Just, D. W., Zaritsky, D., Sand, D. J., Desai, V., \& Rudnick, G. 2010, ApJ, 711, 192

Kauffmann, G., Heckman, T. M., White, S. D. M., et al. 2003, MNRAS, 341,54

Kautsch, S. J., Gonzalez, A. H., Soto, C. A., et al. 2008, ApJL, 688, L5

Kelly, B. C. 2007, ApJ, 665, 1489

Kitaura, F. S., Jasche, J., Li, C., et al. 2009, MNRAS, 400, 183

Kodama, T., Smail, I., Nakata, F., Okamura, S., \& Bower, R. G. 2001, ApJL, 562, L9

Kron, R. G. 1980, ApJS, 43, 305

Lewis, I., Balogh, M., De Propris, R., et al. 2002, MNRAS, 334, 673
Lopes, P. A. A., Ribeiro, A. L. B., \& Rembold, S. B. 2014, MNRAS, 437, 2430

Mamon, G. A., Sanchis, T., Salvador-Solé, E., \& Solanes, J. M. 2004, A\&A, 414, 445

McMillan, P. J. 2011, MNRAS, 414, 2446

Milvang-Jensen, B., Noll, S., Halliday, C., et al. 2008, A\&A, 482, 419

Moran, S. M., Ellis, R. S., Treu, T., et al. 2007, ApJ, 671, 1503

Oemler, A., Jr., Dressler, A., Gladders, M. G., et al. 2013, ApJ, 770, 61

Oman, K. A., Hudson, M. J., \& Behroozi, P. S. 2013, MNRAS, 431, 2307

Owers, M. S., Allen, J. T., Baldry, I., et al. 2017, MNRAS, 468, 1824

Pasquali, A., van den Bosch, F. C., Mo, H. J., Yang, X., \& Somerville, R. 2009, MNRAS, 394, 38

Patel, S. G., Kelson, D. D., Holden, B. P., Franx, M., \& Illingworth, G. D. 2011, ApJ, 735, 53

Pelló, R., Rudnick, G., De Lucia, G., et al. 2009, A\&A, 508, 1173

Peng, Y.-j., Lilly, S. J., Kovač, K., et al. 2010, ApJ, 721, 193

Pimbblet, K. A., Drinkwater, M. J., \& Hawkrigg, M. C. 2004, MNRAS, 354, L61

Poggianti, B. M., De Lucia, G., Varela, J., et al. 2010, MNRAS, 405, 995

Poggianti, B. M., Fasano, G., Bettoni, D., et al. 2009, ApJL, 697, L137

Poggianti, B. M., von der Linden, A., De Lucia, G., et al. 2006, ApJ, 642, 188

Postman, M., \& Geller, M. J. 1984, ApJ, 281, 95

Rines, K., \& Diaferio, A. 2006, AJ, 132, 1275

Rines, K., Geller, M. J., Diaferio, A., \& Kurtz, M. J. 2013, ApJ, 767, 15

Rines, K., Geller, M. J., Kurtz, M. J., \& Diaferio, A. 2003, AJ, 126, 2152

Rudnick, G., von der Linden, A., Pelló, R., et al. 2009, ApJ, 700, 1559

Saglia, R. P., Sánchez-Blázquez, P., Bender, R., et al. 2010, A\&A, 524, A6

Sales, L. V., Navarro, J. F., Abadi, M. G., \& Steinmetz, M. 2007, MNRAS, 379,1475

Schlegel, D. J., Finkbeiner, D. P., \& Davis, M. 1998, ApJ, 500, 525

Serra, A. L., Diaferio, A., Murante, G., \& Borgani, S. 2011, MNRAS, 412, 800

Simard, L., Clowe, D., Desai, V., et al. 2009, A\&A, 508, 1141

Springel, V., White, S. D. M., Jenkins, A., et al. 2005, Natur, 435, 629

Tanaka, M., Finoguenov, A., Kodama, T., et al. 2009, A\&A, 505, L9

Thomas, D., Maraston, C., Schawinski, K., Sarzi, M., \& Silk, J. 2010, MNRAS, 404, 1775

Valentinuzzi, T., Poggianti, B. M., Fasano, G., et al. 2011, A\&A, 536, A34

Vulcani, B., Poggianti, B. M., Oemler, A., et al. 2013, A\&A, 550, A58

Wechsler, R. H., Bullock, J. S., Primack, J. R., Kravtsov, A. V., \& Dekel, A. 2002, ApJ, 568, 52

Wetzel, A. R., Tinker, J. L., Conroy, C., \& van den Bosch, F. C. 2014, MNRAS, 439, 2687

Whiley, I. M., Aragón-Salamanca, A., De Lucia, G., et al. 2008, MNRAS, 387,1253

White, S. D. M., Clowe, D. I., Simard, L., et al. 2005, A\&A, 444, 365

White, S. D. M., \& Zaritsky, D. 1992, ApJ, 394, 1

Zabludoff, A. I., \& Mulchaey, J. S. 1998, ApJ, 496, 39

Zitrin, A., Bartelmann, M., Umetsu, K., Oguri, M., \& Broadhurst, T. 2012, MNRAS, 426, 2944 